\title{
Distributed Implementation of Message Sequence Charts
}

\author{
Rouwaida Abdallah ${ }^{1}$, Loïc Hélouët ${ }^{2}$, Claude Jard ${ }^{1}$ \\ 1 ENS Cachan - IRISA \\ Université Européenne de Bretagne \\ Campus de Ker-Lann, 35170 Bruz, France \\ e-mail: rouwaida.abdallah@bretagne.ens-cachan.fr \\ 2 INRIA - IRISA \\ Campus de Beaulieu, 35042 Rennes, France \\ e-mail: loic.helouet@inria.fr \\ 3 Université de Nantes - LINA \\ 2 rue de la Houssinière, 44322 Nantes, France \\ e-mail: claude.jard@univ-nantes.fr
}

The date of receipt and acceptance will be inserted by the editor

\begin{abstract}
This work revisits the problem of program synthesis from specifications described by High-level Message Sequence Charts. We first show that in the general case, synthesis by a simple projection on each component of the system allows more behaviors in the implementation than in the specification. We then show that differences arise from loss of ordering among messages, and show that behaviors can be preserved by addition of communication controllers, that intercept messages to add stamping information before resending them, and deliver messages to processes in the order described by the specification.
\end{abstract}

Key words scenarios, implementation, distributed system synthesis.

\section{Introduction}

Automatic program synthesis, that is deriving executable code from a highlevel description of a problem (the requirements) is an old dream in computer science. The state of the art progresses through the invention of sophisticated compilation techniques which refine high-level specifications into lower level descriptions that are understandable by an execution platform.

This paper addresses the automatic synthesis problem in the difficult context of distributed applications running on networks of computers. The high-level formalism chosen for this study is Message Sequence Charts (MSCs 
for short) [18]. MSCs have the advantage of specifying interactions patterns between components with a global perspective (such as an external observer) without worrying of how these interactions can be implemented locally by transmission and reception of messages in each component. The lower level for the synthesis is communicating finite automata [9] (CFSMs for short) exchanging messages asynchronously through FIFO channels. This well-known formalism is easily implementable on many distributed platforms built on top of standard communication protocols (TCP, REST, ...).

MSCs is a formal language based on composition of finite communication sequences. It is a prime example of scenario-based languages. It was standardized at the ITU, and has formed the basis for the formal definition of UML sequence diagrams [13]. The approach presented in this paper can certainly be adapted to other languages of this kind such as Use-Case Maps [20] or Live Sequence Charts [14], used for user requirements in distributed systems [4]. Basic MSCs (bMSCs for short) describe finite communication patterns involving a finite set of processes. Communications are supposed point to point and asynchronous. BMSCs are then composed using High-level MSCs (HMSCs for short), a finite automaton whose transitions are labeled by bMSCs. HMSCs are very expressive, and can model infinite state systems. Consequently, the usual verification techniques that apply to finite state machines (model checking of temporal logic formulae, intersecting two specifications to discover undesired behaviors,...) are in general undecidable $[26,10]$.

HMSCs are also difficult to implement. The main difficulty is to transform an HMSC specification with a global view (bMSCs contain complete messages, i.e. a message sending and its corresponding reception appear in the same diagram) of interactions into a set of local views (the communicating machines, which define disjoint sequences of messages sendings and receptions) that is consistent with the original specification. Consistency of an implementation w.r.t a specification can be defined in several ways. In this work, we impose that the synthesized model must exhibit the same behaviors as the original model. In the context of distributed systems modeling, it is natural to define these behaviors as a language of partial orders. Note that all the global coordination expressed by HMSCs can not always be translated to CFSMs. Consequently, some HMSC specifications may not be implementable as CFSMs. For instance, HMSCs allow for the definition of distributed choices, that is configurations in which distinct processes may choose to behave according to different scenarios. The HMSC semantics assumes a global coordination among processes, so all processes decide to execute the same scenario. However, when such distributed choice is implemented by local machines, each process may decide locally to execute a different scenario. When such unspecified situation occurs, the implementation is not consistent with the original HMSC: it exhibits more behaviors and even worse, the synthesized machines can deadlock. This should of course be avoided. HMSCs that do not contain distributed choices are called local HMSCs, and are considered as a reasonnable subclass to target 
a distributed implementation. However, the synthesis solutions proposed so far do not apply to the whole class of local HMSCs.

This paper extends the state of the art by proposing an implementation mechanism for the whole subclass of local message sequence charts, that is HMSC specifications that do not require distributed consensus to be executed. Note that the class of local HMSCs seems a sensible specification model: Indeed, an HMSC which is not local obviously needs additional synchronization messages among participating processes, for instance to reach an agreement on which scenario to execute. One can see this need of additional messages as a transformation of the original non-local HMSC into a local one. Such transformation could be automated, but the solution is not unique. We will not study HMSC localization in this article. The proposed synthesis technique is to project an HMSC on each process participating to the specification. This technique is correct for a subclass of local HMSCs, namely the reconstructible HMSCs, but may produce programs with more behaviors than in the specification for local HMSCs that are not reconstructible [16]. When an HMSC is not reconstructible, we compose the projections with controllers, that intercept messages between processes and tag them with sufficient information to avoid the additional behaviors that appear in the sole projection. The main result of this work is that the projection of the behavior of the controlled system on events of the original processes is equivalent (up to a renaming) to the behavior of the original HMSC. One important aspect of this work is that processes and controllers are independent units of processing, which communicate asynchronously by means of delayed message passing and can be implemented on any distributed architecture. This provides a great genericity of the method.

This paper is organized as follows: Section 2 defines the formal models that will be used in the next sections. Section 3 characterizes the syntactic class of local HMSCs. Section 4 defines the projection operation, that generates communicating finite state machines from an HMSC, and shows that an HMSC and its projection are not equivalent in general. Note that a large part of the material of sections 3 and 4 was already published in our former work [16]. We however include these results in order to obtain a self-contained article. Section 5 proposes a solution based on local control and message tagging to implement properly an HMSC. Section 6 presents a small use case, and section 7 compares our approach with existing techniques, and classifies it with respect to some criteria for scenario-based synthesis approaches. We then conclude and propose future research directions.

\section{Definitions}

In this section we first define our specification model, namely High-level Message Sequence Charts [18]. We then define our implementation model, communicating finite automata. We then list and justify the restrictions that were assumed for the specification model. 


\subsection{Basic MSCs, High-level MSCs}

MSCs are composed of two specification layers: At the lowest level, basic MSCs define interactions among instances, and then these interactions are composed by means of High-level MSCs. A bMSC consists essentially of a finite set of processes (also called instances) denoted by $I$, that run in parallel and exchange messages in a one-to-one, asynchronous fashion. These instances represent different entities of a system such as processes, machines, etc. The life lines of instances are represented as vertical lines. They define sequences of events, ordered from top to bottom. An event can be a message sending or reception, or a local action. Horizontal arrows represent asynchronous messages from one instance to another. An example of bMSC involving three instances $\{$ Sender, Medium, Receiver $\}$, three messages, and an internal action $a$ is shown in Figure 1.

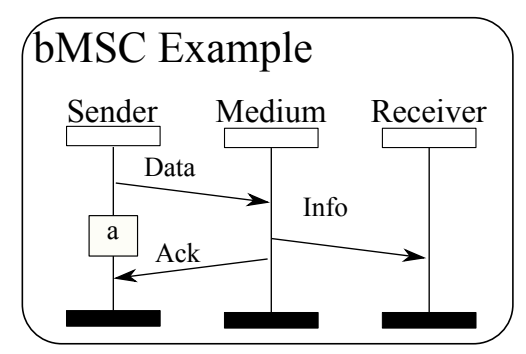

Fig. 1: An example of bMSC

Definition 1 (bMSCs) A bMSC over a finite set of instances $I$ is a tuple $M=(E, \leq, C, \phi, t, \mu)$ where:

$-E$ is a finite set of events. The map $\phi: E \longrightarrow I$ localizes each event on an instance of $I$. $E$ can be split into a disjoint union $\uplus_{p \in I} E_{p}$, where $E_{p}=\{e \in E \mid \phi(e)=p\}$ is the set of events occurring on instance $p$. $E$ can also be considered as the disjoint union $S \uplus R \uplus L$ in order to distinguish send events $(e \in S)$, receive events $(e \in R)$ or local actions $(e \in L)$.

$-C$ is a finite set of message contents and action names.

$-t: E \longrightarrow \Sigma$ gives a type to each event, with

$\Sigma=\{p ! q(m), p ? q(m), a \mid p, q \in I, m, a \in C\}$. We have $t(e)=p ! q(m)$ if $e \in E_{p} \cap S$ is a send event of message " $m$ " from $p$ to $q, t(e)=p ? q(m)$ if $e \in E_{p} \cap R$ is a receive event of message " $m$ " by $p$ from $q$ and $t(e)=a$ if $e \in E_{p} \cap L$ is a local action, named "a" located on $p$.

$-\mu: S \longrightarrow R$ is a bijection that matches send and receive events. If $\mu(e)=f$, then $t(e)=p ! q(m)$ and $t(f)=q ? p(m)$ for some $p, q \in I$ and $m \in C$. 
$-\leq \subseteq E^{2}$ is a partial order relation (the "causal order"). It is required that events of the same instance are totally ordered: $\forall\left(e_{1}, e_{2}\right) \in E^{2} \phi\left(e_{1}\right)=$ $\phi\left(e_{2}\right) \Longrightarrow\left(e_{1} \leq e_{2}\right) \vee\left(e_{2} \leq e_{1}\right)$. For an instance $p$, let us call $\leq_{p}$ this total order. The causal ordering $\leq$ must also reflect the causality induced by the message exchanges, i.e. $\leq=\left(\bigcup_{p \in I} \leq_{p} \cup \mu\right)^{*}$

For a bMSC $M$, we will denote by $\min (M)=\left\{e \in E \mid \forall e^{\prime} \in E, e^{\prime} \leq\right.$ $\left.e \Rightarrow e^{\prime}=e\right\}$, the set of minimal events of $M$. Similarly, we will denote by $\max (M)=\left\{e \in E \mid \forall e^{\prime} \in E, e \leq e^{\prime} \Rightarrow e^{\prime}=e\right\}$ the set of maximal events of $M$. We will call $\phi(E)$ the set of active instances of $M$, and an instance will be called minimal if it carries a minimal event.

BMSCs allow for the compact definition of concurrent behaviors, but are limited to finite and deterministic interactions. To obtain infinite and non-deterministic specifications, we will use High-level MSCs, that compose sequentially bMSCs to obtain languages of bMSCs. Before defining HMSCs, we show how to assemble two bMSCs:

Definition 2 (Sequential composition) Let $M_{1}=\left(E_{1}, \leq_{1}, C_{1}, \phi_{1}, t_{1}, \mu_{1}\right)$ and $M_{2}=\left(E_{2}, \leq_{2}, C_{2}, \phi_{2}, t_{2}, \mu_{2}\right)$ be two bMSCs, defined over disjoint sets of events. The sequential composition of $M_{1}$ and $M_{2}$ is denoted by $M_{1} \circ M_{2}$. It consists in a concatenation of the two bMSCs instance by instance, and is obtained as follows. $M_{1} \circ M_{2}=(E, \leq, C, \phi, t, \mu)$, where:

$$
\begin{aligned}
& -E=E_{1} \cup E_{2}, C=C_{1} \cup C_{2} \\
& -\forall e, e^{\prime} \in E, e \leq e^{\prime} \text { iff } e \leq_{1} e^{\prime} \text { or } e \leq_{2} e^{\prime} \text { or } \exists\left(e_{1}, e_{2}\right) \in E_{1} \times E_{2}: \phi_{1}\left(e_{1}\right)= \\
& \quad \phi_{2}\left(e_{2}\right) \wedge e \leq_{1} e_{1} \wedge e_{2} \leq_{2} e^{\prime} \\
& -\forall e \in E_{1}, \phi(e)=\phi_{1}(e), \mu(e)=\mu_{1}(e), t(e)=t_{1}(e) \\
& -\forall e \in E_{2}, \phi(e)=\phi_{2}(e), \mu(e)=\mu_{2}(e), t(e)=t_{2}(e)
\end{aligned}
$$

Note that the definition requires the concatenated bMSCs to be defined over disjoint sets of events. In the rest of the paper, we will use concatenation to assemble several occurrences of the same bMSC. Slightly abusing the definition, we will consider that concatenation $M_{1} \circ M_{2}$ is always defined, and if $E_{1} \cap E_{2} \neq \emptyset$, we will consider that $M_{1} \circ M_{2}$ is a bMSC obtained by composing $M_{1}$ with an isomorphic copy of $M_{2}$ defined over a set of events that is disjoint from $E_{1}$. In particular, this allows us to define, for a bMSC $M$, the bMSC $M \circ M$ which denotes a scenario with two consecutive occurrences of $M$. An intuitive and graphical interpretation for $M_{1} \circ M_{2}$ is that the interactions in $M_{2}$ are appended to $M_{1}$ after $M_{1}$ (i.e. drawn below $M_{1}$ ). An example of sequential composition is shown in Figure 2: The bMSC $M_{1} \circ M_{2}$ can simply be obtained by drawing $M_{2}$ below $M_{1}$, and extending the lifelines of instances. Note that sequential composition does not require both bMSCs to have the same set of instances.

HMSC diagrams are automata that compose bMSCs or other HMSCs. This allows for the definition of iterations and choices. We will suppose without loss of generality that our HMSCs comprise only one hierarchical level, i.e. they are automata whose transitions are labeled by bMSCs. 


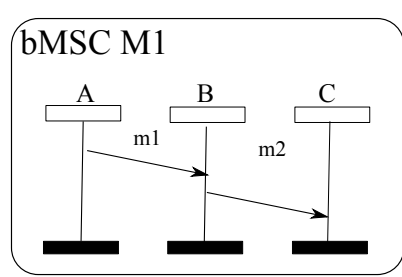

(a)

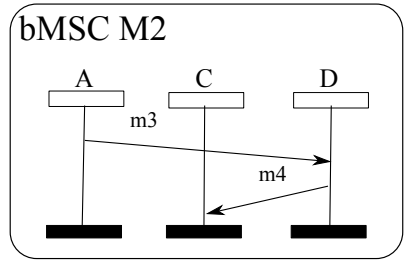

(b)

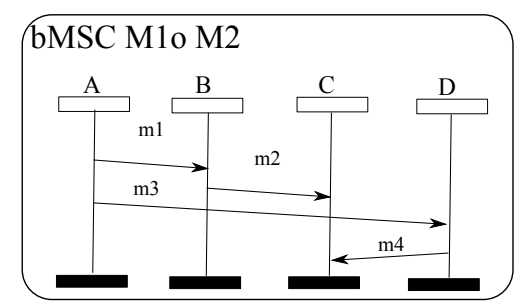

(c)

Fig. 2: Two bMSCs $M_{1}$ and $M_{2}$ and their concatenation $M_{1} \circ M_{2}$

Definition 3 (HMSCs) An HMSC is a graph $H=\left(I, N, \rightarrow, \mathcal{M}, n_{0}\right)$, where

- I is a finite set of instances.

$-N$ is a finite set of nodes and $n_{0} \in N$ is the initial node of $H$.

$-\mathcal{M}$ is a finite set of bMSCs which participating instances belong to I, and defined on disjoint sets of events.

$-\rightarrow \subseteq N \times \mathcal{M} \times N$ is the transition relation .

HMSCs contain a unique initial node $n_{0}$, that has no incoming transition (i.e, there is no transition of the form $\left(n, M, n_{0}\right)$ in $\rightarrow$ ), but also sink nodes, i.e. nodes that have no successor, and choice nodes, i.e. nodes that have several successors. Figure 3 shows an example of HMSC with two nodes $n_{0}, n_{1}$, where $n_{0}$ is the initial node (and also a choice node), and $n_{1}$ is a sink node. A node is represented by a circle. Initial nodes have a downward pointing triangle connected to them, and sink nodes are connected to an upward pointing triangle. In this example, we have $\mathcal{M}=\left\{M_{1}, M_{2}\right\}$ where $M_{1}, M_{2}$ are the bMSCs defined in Figure 2. The transition relation contains two transitions, namely $\left(n_{0}, M_{1}, n_{0}\right)$ and $\left(n_{0}, M_{2}, n_{1}\right)$. In the example of Figure 3 , the behavior $M_{1}$ can be repeated an arbitrary number of times, and then be followed by the behavior described in $M_{2}$.

For convenience, we will consider that all nodes, except possibly the initial node and sink nodes are choice nodes, i.e. have several successors by the transition relation. This results in no loss of generality, as an HMSC can be always transformed in such a canonical form by concatenating bMSCs appearing in a path. A transition from a (choice) node will be frequently called $a$ branch of this choice. We also require HMSCs to be deterministic, 
that is if $\left(n, M_{1}, n_{1}\right) \in \longrightarrow \wedge\left(n, M_{2}, n_{2}\right) \in \longrightarrow$, then $M_{1} \neq M_{2}$. This can be ensured by the standard determinization procedure of finite automata.

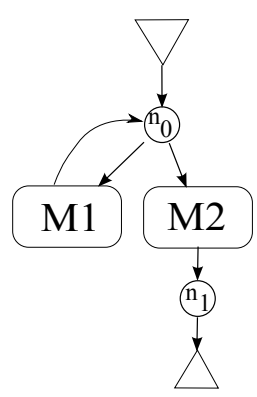

(a)

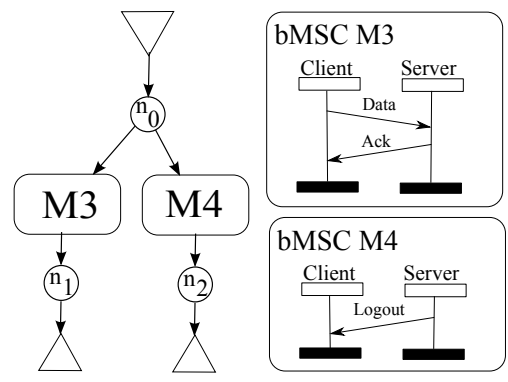

(b)

Fig. 3: An example of local HMSC $a$ ) and a non-local HMSC $b$ )

\subsection{Semantics of HMSCs}

In the next sections, executions of bMSCs will be represented as partially ordered multisets of events (pomsets). These pomsets are not necessarily bMSCs, as we will consider incomplete executions in which some messages have been sent and not yet received. This notion of incomplete execution is captured by the definition of pieces and prefixes.

Definition 4 (prefix, suffix, piece of bMSCs) Let $M=(E, \leq, C, \phi, t, \mu)$ be a bMSC. A prefix of $M$ is a tuple $\left(E^{\prime}, \leq^{\prime}, C^{\prime}, \phi^{\prime}, t^{\prime}, \mu^{\prime}\right)$ such that $E^{\prime}$ is a subset of $E$ closed by causal precedence (i.e. $e \in E^{\prime} \wedge f \leq e \Longrightarrow f \in$ $\left.E^{\prime}\right)$ and $\leq^{\prime}, C^{\prime}, \phi^{\prime}, t^{\prime}, \mu^{\prime}$ are restrictions of $\leq, C, \phi, t, \mu$ to $E^{\prime}$. A suffix of $M$ is a tuple $\left(E^{\prime}, \leq^{\prime}, C^{\prime}, \phi^{\prime}, t^{\prime}, \mu^{\prime}\right)$ such that $E^{\prime}$ is closed by causal succession (i.e. $e \in E^{\prime} \wedge e \leq f \Longrightarrow f \in E^{\prime}$ ) and $\leq^{\prime}, C^{\prime}, \phi^{\prime}, t^{\prime}, \mu^{\prime}$ are restrictions of $\leq, C, \phi, t, \mu$ to $E^{\prime}$. A piece of $M$ is the restriction of $M$ to a set of events $E^{\prime}=E \backslash X \backslash Y$, such that the restriction of $M$ to $X$ is a prefix of $M$ and the restriction of $M$ to $Y$ is a suffix of $M$.

Note that prefixes, suffixes and pieces are not always bMSCs, as their message mappings $m$ are not necessarily bijections from sending events to receiving events. In the rest of the paper, we will denote by $\operatorname{Pref}(M)$ the set of all prefixes of a bMSC $M$. We will denote by $O_{\epsilon}$ the empty prefix, i.e. the prefix that contains no event. For a particular type of action $a$, we will denote by $O_{a}$ a piece containing a single event of type $a$. The examples of Figure 4 shows a bMSC $M$ involving three processes $P, Q, R$, a prefix $\operatorname{Pr}$, a suffix $S$, and a piece $P c$. Observe that $P c$ is obtained by erasing $P r$ and $S$ from $M$. Note also that $P r, S$ and $P c$ contain incomplete messages. 


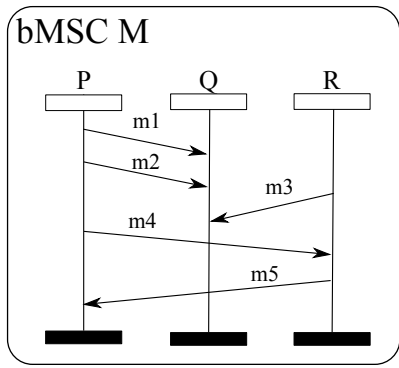

(a)

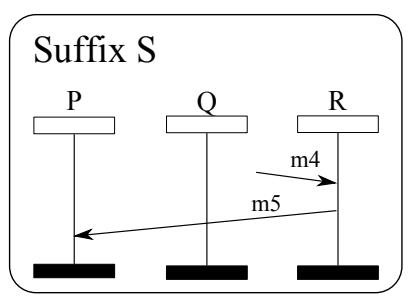

(c)

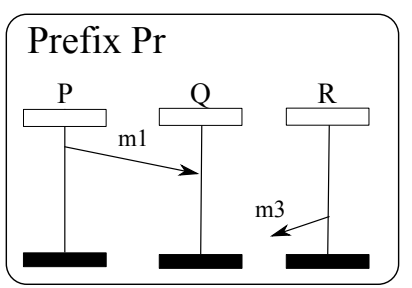

(b)

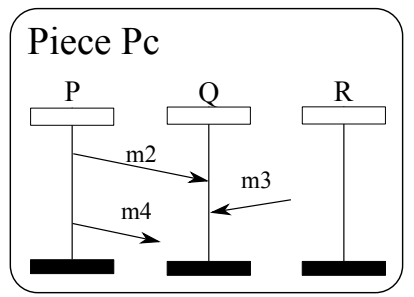

(d)

Fig. 4: A bMSC (a), a prefix (b), a suffix (c) and a piece (d)

In the next sections, we will also need to concatenate prefixes and pieces of bMSCs. Prefix and piece concatenation is defined alike bMSC concatenation with an additional phase that rebuilds the message mappings. Let $O_{1}$ be a prefix of a bMSC, and $O_{2}$ be a piece of bMSC. Then, the concatenation of $O_{1}$ and $O_{2}$ is denoted by $O_{1} \circ O_{2}=(E, \leq, C, \phi, t, \mu)$, where $E, \leq, C, \phi$, and $t$ are defined as in definition 2 and $\mu$ is a function that associates the $n^{\text {th }}$ sending event from $p$ to $q$ to the $n^{\text {th }}$ reception from $p$ on $q$ for every pair of processes $p, q \in I$. Note that this sequencing is not defined if for some $p, q, n$, the types of the $n^{t h}$ sending and reception do not match, that is one event is of the form $p ! q(m)$ and the other one $q ? p\left(m^{\prime}\right)$ with $m \neq m^{\prime}$. In particular, we will denote by $O \circ\{e\}$ the prefix obtained by concatenation of a single event $e$ to a prefix $O$.

Definition 5 (HMSC behavior) Let $H=\left(I, N, \rightarrow, \mathcal{M}, n_{0}\right)$ be an HMSC. $A$ path of $H$ is a sequence $\rho=\left(n_{0}, M_{0}, n_{1}\right)\left(n_{1}, M_{1}, n_{2}\right) \ldots\left(n_{k}, M_{k}, n_{k+1}\right)$ of transitions. We will say that a path is acyclic if and only if it does not contain the same transition twice. We define as Paths $(H)$ the set of paths of $H$ starting from the initial node. A path $\rho=\left(n_{0}, M_{0}, n_{1}\right) \ldots\left(n_{k}, M_{k}, n_{k+1}\right)$ in Paths $(H)$ defines a sequence $M_{0} \cdot M_{1} \ldots M_{k} \in \mathcal{M}^{*}$ of bMSCs. We will denote by $O_{\rho}$ the bMSC associated to $\rho$ and define it as $O_{\rho}=M_{0} \circ M_{1} \circ \cdots \circ M_{k}$. The language of $H$ is the set of behaviors $\mathcal{L}(H)=\bigcup_{\rho \in \operatorname{Paths}(H)} \operatorname{Pref}\left(O_{\rho}\right)$.

To simplify notation, we will write $\rho=n_{0} \stackrel{M_{0}}{\longrightarrow} n_{1} \stackrel{M_{1}}{\longrightarrow} n_{2} \ldots \stackrel{M_{k}}{\longrightarrow} n_{k+1}$ to denote a path $\rho=\left(n_{0}, M_{0}, n_{1}\right)\left(n_{1}, M_{1}, n_{2}\right) \ldots\left(n_{k}, M_{k}, n_{k+1}\right)$. Note that 
our definition of the language of an HMSC $H$ includes all prefixes of bMSCs generated by $H$. Note also that the language of an HMSC is not a regular language in general. In the example of Figure 3, for instance, behaviors in which machine $A$ asynchronously sends an arbitrary number of messages without waiting for their reception are contained in the language of the specification. Clearly, such behaviors can not be represented as a regular language. A correct implementation of an HMSC $H$ is a distributed system reproducing exactly (and nothing more) $\mathcal{L}(H)$.

\subsection{Communicating Finite State Machines}

In this section, we introduce our implementation model, namely Communicating Finite State Machines (CFSM) [9]. A CFSM $\mathcal{A}$ is a network of finite state machines that communicate over unbounded, non-lossy, error-free and FIFO communication channels. Before formally introducing CFSM, let us justify the choice of this model as implementation formalism. First of all, HMSCs describe the behavior of independent agents, which continuously run sequences of communication events. Hence, if we want to respect the independence of agents in the architecture depicted in an HMSC, we need a target model that allows for the definition of parallel components. Models such as Petri nets or networks of automata communicating via shared actions fulfill these requirements. However, it is clearly stated in the Z.120 standard [18] that bMSCs and HMSCs depict the behavior of agents that communicate asynchronously, which rules out communications using shared actions. One can also notice that the projection of an HMSC on a single instance gives a regular language. Last, one shall notice that HMSCs semantics can enforce messages between a pair of processes to respect FIFO ordering, which can not be enforced by Petri nets. In fact, it has been shown that synthesis of Petri nets from HMSCs usually produces an overapproximation of the initial HMSC language [10]. All these considerations call for the use of CFSMs as target architecture.

We will write $\mathcal{A}=\|_{i \in I} A_{i}$ to denote that $\mathcal{A}$ is a network of machines describing the behaviors of a set of machines $\left\{A_{i}\right\}_{i \in I}$. A communication buffer $B_{(i, j)}$ is associated to each pair of instances $(p, q) \in I^{2}$. Buffers will implement messages exchanges defined in the original HMSC. More formally, we can define a communicating automaton as follows:

Definition $6 \mathrm{~A}$ communicating automaton associated to an instance $\mathrm{p}$ is a tuple $A_{p}=\left(Q_{p}, \delta_{p}, \Sigma_{p}, q_{0, p}\right)$ where $Q_{p}$ is a set of states, $q_{0, p}$ is the initial state, $\Sigma_{p}$ is an alphabet with all letters of the form $p ! q(m) p ? q(m)$ or a, symbolizing message sending to a process $q$, reception from a process $q$, an atomic action a executed by process $p$, or a silent move $\epsilon$. The transition relation $\delta_{p} \subseteq Q_{p} \times \Sigma_{p} \times Q_{p}$ is composed of triples $\left(q, \sigma, q^{\prime}\right)$ indicating that the machine moves from state $q$ to state $q^{\prime}$ when executing action $\sigma$. A CFSM $\mathcal{A}=\|_{i \in I} A_{i}$ is a composition of communicating automata. 


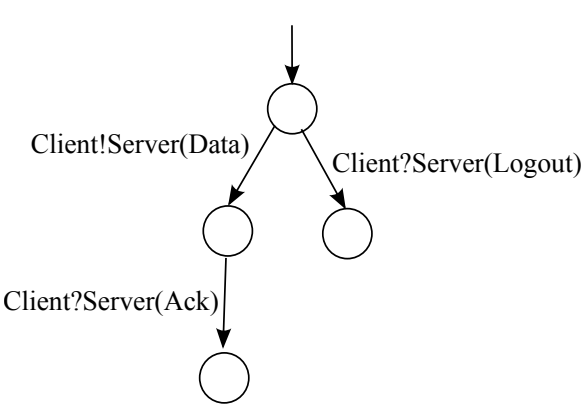

$\mathrm{A}_{\text {Client }}$

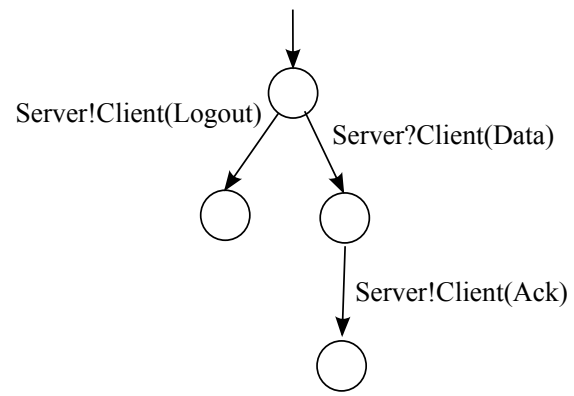

A

Fig. 5: Two communicating machines

Figure 5 describes a CFSM composed of two finite state machines $A_{\text {Client }}$ and $A_{\text {Server }}$. The initial states of these two machines are denoted by a dark incoming arrow. Each run of a set of communicating machines defines a prefix, that can be built incrementally starting from the empty prefix, and appending one executed event after the other (i.e. it is built from a total ordering of all events occurring on the same process, plus a pairing of messages sendings and receptions). Then, the language $\mathcal{L}(\mathcal{A})$ of a set of communicating machines is the set of all prefixes associated to runs of $\mathcal{A}$.

The semantics of CFSM is usually defined as sequences of events. Each event occurs on a single process, and changes the configuration of the CFSM. A configuration of a network of automata $\mathcal{A}=\|\|_{i \in I}$ is a pair $C=(L, W)$ where $L$ is a sequence of states $q_{1} \ldots q_{I}$ depicting the local state of each communicating machine, and $W=\left\{w_{11}, \ldots w_{1|I|}, w_{21}, \ldots w_{2|I|}, \ldots w_{|I||I|}\right\}$ is a set of $|I|^{2}$ words depicting the contents of message buffers. Each $w_{i j}$ is a sequence of message names, and depicts the contents of the queue from $A_{i}$ to $A_{j}$. Then, the behavior of $\mathcal{A}$ is defined as follows:

- all machines start from their initial states with all communication buffers empty, that is the initial configuration is $C_{0}=\left(L_{0}=q_{0,1} \ldots q_{0,|I|}, W_{0}=\right.$ $\{\epsilon, \ldots \epsilon\})$.

- From a configuration $C$, a machine $A_{p}$ can send a message $m$ to a machine $A_{q}$ if $A_{p}$ is in local state $q_{p}$, there exists a transition $\left(q_{p}, p ! q(m), q_{p}^{\prime}\right)$ in $A_{p}$. Executing this action $p ! q(m)$ simply appends $m$ to the buffer $w_{p, q}$ from $p$ to $q$ and changes $A_{p}$ 's local state to $q_{p}^{\prime}$ in the configuration. Hence, if $C=(L, W)$ with $L=q_{0} \ldots q_{p} \ldots q_{|I|}$ and $W=$ $\left\{w_{11}, \ldots w_{p, q} \ldots w_{|I||I|}\right\}$, executing $p ! q(m)$ results in a configuration $C^{\prime}=$ $\left(L^{\prime}, W^{\prime}\right)$ with $L^{\prime}=q_{0} \ldots q_{p}^{\prime} \ldots q_{|I|}$ and $W^{\prime}=\left\{w_{11}, \ldots w_{p, q} . m \ldots w_{|I||I|}\right\}$ Local actions of communicating automata change the local state of a machine and leave the buffer contents unchanged.

- From a configuration $C, A_{p}$ can receive a message $m$ from process $q$, if $A_{p}$ is in local state $q_{p}$, there exists a transition $\left(q_{p}, p ? q(m), q_{p}^{\prime}\right)$ in $A_{p}$, 
and the first letter of $w_{q, p}$ is $m$ (which means that $m$ is the first message that has to be received in the queue from $q$ to $p$ ). Executing this action $p ? q(m)$ simply removes $m$ from the buffer $w_{p, q}$ from $p$ to $q$ and changes $A_{p}$ 's local state to $q_{p}^{\prime}$ in the configuration. Hence, if $C=(L, W)$ with $L=q_{0} \ldots q_{p} \ldots q_{|I|}$ and $W=\left\{w_{1} 1, \ldots w_{p, q}=m . w \ldots w_{|I||I|}\right\}$, executing $p ? q(m)$ results in a configuration $C^{\prime}=\left(L^{\prime}, W^{\prime}\right)$ with $L^{\prime}=q_{0} \ldots q_{p}^{\prime} \ldots q_{|I|}$ and $W^{\prime}=\left\{w_{11}, \ldots w_{p, q}=w \ldots w_{|I||I|}\right\}$.

This way, CFSMs define sequences of actions $\sigma_{1} \ldots \sigma_{k}$ that can be executed by their local components from their initial states. Each action moves the communicating machines from one configuration to another. However, CFSM are concurrent models, and their executions can be represented in a non-interleaved way by bMSC prefixes.

Definition 7 Let $\mathcal{A}=\| A_{i \in I} A_{i}$ be a CFSM. The language of $\mathcal{A}$ is denoted by $\mathcal{L}(\mathcal{A})$ and is the set of prefixes defined inductively as follows :

- the prefix associated to an empty sequence of actions is the empty prefix $O_{\epsilon}$,

- the prefix associated to a sequence of actions $\sigma_{1} \ldots \sigma_{k} . \sigma_{k+1}$ of $\mathcal{A}$ is the prefix $O \circ\{e\}$ where $e$ is an event labeled by $\sigma_{k+1}$ and $O$ is the prefix associated to $\sigma_{1} \ldots \sigma_{k}$.

\subsection{Restrictions}

We have assumed some restrictions on the scenarios that we implement. Some of them are introduced for the sake of readability, and some of them are essential to ensure a solution to the synthesis problem. Standard notation of bMSCs allow for the definition of a zone on an instance axis called co-region. Events appearing in a co-region can be executed in any order. We do not consider co-regions, but they can be simulated by adding to an HMSC a finite number of alternatives enumerating all possible interleavings of events. We also consider that HMSCs are deterministic, and that two bMSCs labeling distinct transitions of a local HMSC start with distinct messages. We use this assumption to differentiate branches at runtime. We could achieve a similar result by introducing additional tags during synthesis. However, this mild restriction simplifies the notations and proofs.

BMSCs also allow behaviors with message overtaking, i.e. in which some messages mandatorily cross other messages from the same bMSC. In this paper, we consider only FIFO architectures as a target for synthesis. This is hence a natural restriction to consider that all bMSCs are FIFO, that is for two sending events $e, e^{\prime}$ such that $p=\phi(e)=\phi\left(e^{\prime}\right), q=\phi(\mu(e))=\phi\left(\mu\left(e^{\prime}\right)\right)$ we always have $e \leq_{p} e^{\prime} \Longleftrightarrow \mu(e) \leq_{q} \mu\left(e^{\prime}\right)$. Note that our synthesis technique could be easily adapted to allow overtaking in bMSCs. This requires a slight modification of the communication architecture, to allow a bounded lookahead at the contents of communication buffers, and consumption of 
messages appearing at a fixed position in a FIFO buffer rather that in first position. Such semantics exists for instance in extended automata models such as SDL, and a synthesis technique to generate SDL code from HMSCs in which bMSCs contain message crossings was proposed in [1].

We restrict to HMSCs without parallel frames for deeper reasons. When parallel frames are used, the behavior of an agent may not be a regular language, i.e. it may not be expressible as a finite state machine. The implementation technique proposed in this paper uses vectorial clocks that may grow unboundedly, but the systems generated always comport a finite number of control states. Furthermore, the use of parallel frames may add a new source of unexpected behaviors, as one agent may have to react differently when a pair of actions $a, b$ are executed concurrently or in sequence, and such non-determinism may lead to the execution of unspecified behaviors. Hence, we doubt that a simple machine model can handle at the same time unbounded parallelism in agents and asynchronous communications, to implement the extremely complex (and very often ambiguous) behaviors allowed with parallel frames. Such extension to CFSMs goes beyond the scope of this paper.

\section{Local HMSCs}

Consider a choice node in an HMSC, that is a node $n$ with at least two outgoing transitions $\left(n, M_{1}, n_{1}\right)$ and $\left(n, M_{2}, n_{2}\right)$. Executing an event in $M_{1}$ (resp. $M_{2}$ ) can be seen as taking the decision to execute the whole behavior contained in $M_{1}$ (resp. $\left.M_{2}\right)$. Once the decision to perform $M_{1}$ or $M_{2}$ is taken, all the other instances have to conform to this decision to remain consistent with the HMSC specification. Hence, every bMSC $M_{i}$ labeling a transition leaving a choice node defines a set of deciding instances $\phi\left(\operatorname{Min}\left(M_{i}\right)\right)$, which is the set of instances that carry the minimal events of $M_{i}$, and hence can take the decision to perform bMSC $M_{i}$. Obviously, the minimal events in each $M_{i}$ cannot be message receptions.

We can now state the main difficulty when moving from HMSCs to local machines. In an HMSC, the possible executions are built by concatenating bMSCs one after another. Hence in an execution of an HMSC, all processes conform to a single sequence of bMSCs collected along a path. In a CFSM setting, when two processes have to take a decision to perform scenario $M_{1}$ or $M_{2}$, they can of course take concurrently the same decision, but conversely, one instance can decide to perform scenario $M_{1}$ while the other instance decides to perform $M_{2}$. Consider for instance the HMSC of Figure 3-b. The instance Client can decide to send Data and wait for an acknowledgment while the instance Server decides to send Logout. Such situation can lead to a deadlock of the system. Even worse, this scenario was not specified in the original description. Such unspecified scenarios are frequently called "implied scenarios", and were originally studied in [30]. The main intuition behind this notion of implied scenario is that even though a 
scenario was not part of the original specification $H$, as a distributed implementation of $H$ can execute it, then it should be considered as part of the specification, and explicitly appended to the original model [31]. This approach may work for simple cases, but not for all kinds of HMSCs. First of all, an HMSC may exhibit an infinite number of implied scenarios. Furthermore, it is undecidable if an implied scenario is a prefix of some run that already exists in the original specification (this problem can be brought back to a language inclusion problem for HMSCs, which was shown to be undecidable $[26,10])$. So, one can not decide if a specification already includes an implied scenario that was discovered for a particular choice node. Furthermore, every implied behavior appended to an HMSC may produce new implied scenarios and the growth of a specification due to the integration of these new behaviors may never stop. A safer design choice is to consider that situations leading to non-local choices and hence to implied scenarios have to be avoided. For this, we define local HMSCs.

When the outgoing transitions of a choice node are labeled by bMSCs with distinct deciding instances, then, without additional synchronization the synthesized machines might decide to perform distinct scenarios. This situation is called non-local choice, and should be avoided in a specification. We consider that specifications containing non-local choices are not refined enough to be implemented.

Definition 8 (Local choice node) Let $H=\left(I, N, \rightarrow, \mathcal{M}, n_{0}\right)$ be an HMSC, and let $c \in N$ be a choice node of $H$. Choice $c$ is local if and only if for every pair of (not necessarily distinct) paths $\rho=c \stackrel{M_{1}}{\longrightarrow} n_{1} \stackrel{M_{2}}{\longrightarrow} n_{2} \ldots n_{k}$ and $\rho^{\prime}=c \stackrel{M_{1}^{\prime}}{\longrightarrow} n_{1} \stackrel{M_{2}^{\prime}}{\longrightarrow} n_{2}^{\prime} \ldots n_{k}^{\prime}$ there is a single minimal instance in $O_{\rho}$ and in $O_{\rho^{\prime}}$ (i.e. $\phi\left(\operatorname{Min}\left(O_{\rho}\right)\right)=\phi\left(\operatorname{Min}\left(O_{\rho^{\prime}}\right)\right)$ and $\left.\left|\phi\left(\operatorname{Min}\left(O_{\rho}\right)\right)\right|=1\right)$. $H$ is called a local HMSC if all its choices are local.

We will also say that an HMSC is non-local if one of its choices is not local. Intuitively, the locality property described in [6] guarantees that every choice is controlled by a unique instance. We will show however that ensuring locality of choices is not sufficient to guarantee a correct synthesis.

Proposition 1 (Deciding locality) Let $H$ be an HMSC. $H$ is not local iff there exists a node $c$ and a pair of acyclic paths $\rho, \rho^{\prime}$ originating from $c$, such that $O_{\rho}$ and $O_{\rho^{\prime}}$ have more than one minimal instance.

Proof: One direction is straightforward: If we can find a node $c$ and two (acyclic) paths with more than one deciding instance, then obviously, $c$ is not a local choice, and $H$ is not local. Let us suppose now that for every node $c$, and for every pair of acyclic paths of $H$ originating from $c$, we have only one deciding instance. Now, let us suppose that there exists a node $c_{1}$ and two paths $\rho_{1}, \rho_{1}^{\prime}$ such that at least one (say $\rho_{1}$ ) of them is not acyclic, and ends with transitions that appear several times along this path. Then $\rho_{1}$ has a finite acyclic prefix $w_{1}$. The set of minimal instances in $O_{w 1}$ and in $O_{\rho 1}$ is the same, as for all bMSCs $M, \phi(\min (M \circ M))=\phi(\min (M))$. 
Hence, $c, \rho_{1}, \rho_{1}^{\prime}$ are witnesses for the non-locality of $H$ iff $c, w_{1}, \rho_{1}^{\prime}$ are also such witnesses.

Theorem 1 (Complexity of local choices) Deciding if an HMSC is local is in $c o-N P$.

Proof: The objective is to find a counter example, that is two paths originating from the same node with distinct deciding instances. One can choose in linear time in the size of $H$ a node $c$ and two finite acyclic paths $\rho_{1}, \rho_{2}$ of $H$ starting from $c$, that is sequences of bMSCs of the form $M_{1} \ldots M_{k}$. One can compute a concatenation $O=M_{1} \circ \cdots \circ M_{k}$ in polynomial time in the total size of the ordering relations. Note that to compute minimal events of a sequencing of two bMSCs, one does not have to compute the whole causal ordering $\leq$, and only has to ensure that maximal and minimal events on each instance in two concatenated bMSCs are ordered in the resulting concatenation. Hence it is sufficient to recall a covering of the local ordering $\leq_{p}$ on each process $p \in I$ plus the message relation $m$. Then finding the minimal events (or equivalently the minimal instances) of $O$ can also be performed in polynomial time in the number of events of $O$, as $\operatorname{Min}(M)=E \backslash\left\{f \mid \exists e, e \leq_{p} f \vee f=\mu(e)\right\}$.

From theorem 1, an algorithm that checks locality of HMSCs is straightforward. It consists in a width first traversal of acyclic paths starting from each node of the HMSC. If at some time we find two paths with more than one minimal instance, then the choice from which these paths start is not local. Note that the set of minimal instances on a path $\rho$ (or the whole bMSC $O_{\rho}$ labeling this path) needs not be recomputed everytime a path is extended, and can be updated at the same time as paths. Indeed, if $\rho=\rho_{1}$. $\rho_{2}$ is a path of $H$, then $\phi\left(\operatorname{Min}\left(M_{\rho}\right)\right)=\phi\left(\operatorname{Min}\left(M_{\rho_{1}}\right)\right) \cup\left(\phi\left(\operatorname{Min}\left(M_{\rho_{2}}\right)\right) \backslash \phi\left(M_{\rho_{1}}\right)\right)$. It is then sufficient for each path to maintain the set of instances that appear along this path, and the set of minimal instances, without memorizing exactly the scenario that is investigated.

Algorithm 1 was originally proposed in [16]. It builds a set of acyclic paths starting from each node of an HMSC. A non-local choice is detected if there is more than one deciding instance for a node $c$. The algorithm remembers a set of acyclic paths $P$, extends all of its members with new transitions when possible, and places a path $\rho$ in $M A P$ as soon as the set of transitions used in $\rho$ contains a cycle. The correctness of the algorithm is guaranteed by theorem 1 , and as we consider a finite set of maximal acyclic paths, termination is guaranteed.

\section{The Synthesis Problem}

The objective of the synthesis algorithm from an HMSC $H$ is to obtain a $\operatorname{CFSM} \mathcal{A}$ that behaves exactly as $H$. An obvious solution is to project the original HMSC on each instance, that is if $H$ is defined over a set of instances $I$, we want to build a $\operatorname{CFSM} \mathcal{A}=\|_{i \in I} A_{i}$ such that $\mathcal{L}(H)=\mathcal{L}(\mathcal{A})$. 


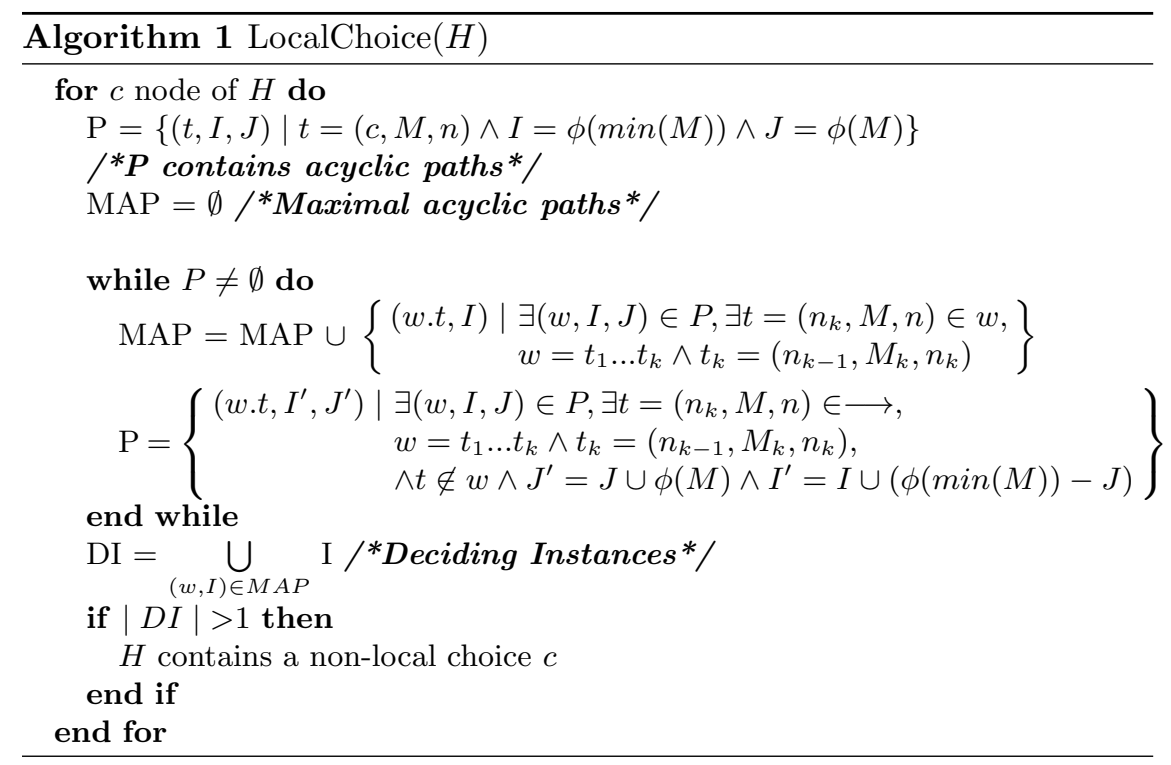

The principle of projection is to copy the original HMSC on each instance, and to remove all the events that do not belong to the considered instance. This operation preserves the structure of the HMSC automaton: Starting from an automaton labeled by bMSCs, we obtain an automaton labeled by (possibly empty) sequences of events located on the considered instance. This object can be considered as a finite state automaton by adding intermediary states in sequences of events. Empty transitions can be removed by the usual $\varepsilon$-closure procedure for finite state automata (see for instance chapter 2.4 of [17]).

Definition 9 (Projection) Let us consider an $H M S C H=\left(I, N, \rightarrow, \mathcal{M}, n_{0}\right)$. The set of events of a bMSC $M$ is denoted by $E_{M}$, and the set of events of $M$ located on instance $i$ by $E_{M i}$. The set $E_{M_{i}}$ is totally ordered by $\leq_{i}$. We denote its elements by $e_{1}, \cdots, e_{\left|E_{M i}\right|}$. The finite state automaton $A_{i}$, result of the projection of $H$ onto the instance $i$ is $A_{i}=\left(Q_{i}, \rightarrow_{i}, E_{i} \cup\{\varepsilon\}, n_{0}\right)$. We encode states of $A_{i}$ as tuples $\left(n, M, n^{\prime}, k\right) \in N \times \mathcal{M} \times N \times \mathbb{N}$, where the first three components designate an HMSC transition labeled by a bMSC $M$ defined over a set of events $E_{M}$, and the last component $k$ is an index ranging from 1 to $\left|E_{M_{i}}\right|$ indicating the progress of instance $i$ during $M$, or simply as a reference to an HMSC node $n$ (designating a configuration in which $A_{i}$ has not yet started the execution of a bMSC from $n)$. We then have $Q_{i}=\{n\} \cup\left\{\left(n, M, n^{\prime}, k\right)\left|\left(n, M, n^{\prime}\right) \in \longrightarrow \wedge<\right| E_{M_{i}} \mid\right\}$, 
and $E_{i}=\bigcup_{M \in \mathcal{M}} E_{M i}$. We can then define the transition relation $\longrightarrow_{i}$ as $\longrightarrow{ }_{i}=\left\{\left(n, \epsilon, n^{\prime}\right)\left|\exists\left(n, M, n^{\prime}\right) \in \longrightarrow \wedge\right| E_{M_{i}} \mid=0\right\}$

$\cup\left\{\left(n, t\left(e_{1}\right), n^{\prime}\right)\left|\exists\left(n, M, n^{\prime}\right) \in \longrightarrow \wedge\right| E_{M_{i}} \mid=1\right\}$

$\cup\left\{\left(n, t\left(e_{1}\right),\left(n, M, n^{\prime}, 1\right)\right)\left|\left(n, M, n^{\prime}\right) \in \longrightarrow \wedge\right| E_{M_{i}} \mid \geq 2\right\}$

$\cup\left\{\left(\left(n, M, n^{\prime}, k-1\right), t\left(e_{k}\right),\left(n, M, n^{\prime}, k\right)\right)\left|\left(n, M, n^{\prime}\right) \in \longrightarrow \wedge 2 \leq k<\right| E_{M_{i}} \mid\right\}$

$\cup\left\{\left(\left(n, M, n^{\prime}, k-1\right), t\left(e_{k}\right), n^{\prime}\right)\left|\left(n, M, n^{\prime}\right) \in \longrightarrow \wedge k=\right| E_{M_{i}} \mid\right\}$
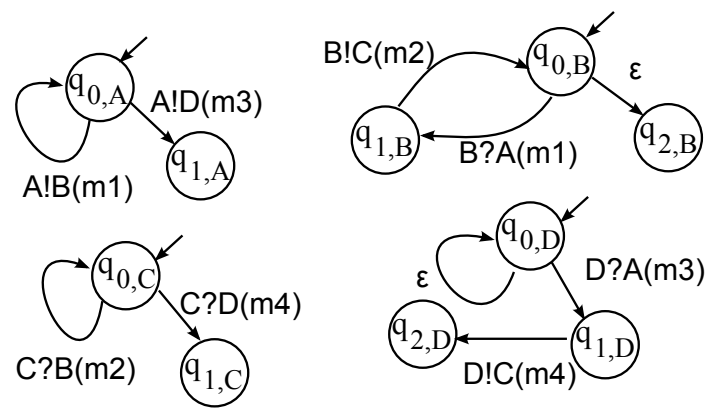

Fig. 6: The instance automata projected from the HMSC of Fig. 3-a).

The synthesis by projection from the HMSC of Figure 3-a) produces the CFSM of Figure 6 . Note that as instance $D$ is not active in bMSC $M 1$, there is an $\epsilon$-transition in the automaton associated to $D$. The synthesis from the HMSC of Figure 3-b) produces the CFSM of Figure 5. In this model, the CFSM can behave as specified in scenarios $M_{1}$ and $M_{2}$. However, $A_{\text {client }}$ can also decide to send a Data message while $A_{\text {Server }}$ sends a logout message. This situation was not specified in the HMSC of Figure 3-b), so the CFSM of Figure 5 cannot be considered as a correct implementation. In general, the projection of an HMSC on its instances can define more behaviors than the original specification, but can also deadlock. Hence, synthesis by projection on instance is not correct for any kind of HMSC. It was proved in [16] that the synthesized language contains all runs of the HMSC specification.

Theorem 2 ([16]) Let $H$ be an HMSC and let $\mathcal{A}$ be the CFSM obtained by projection of $H$ on its instances. Then $\mathcal{L}(H) \subseteq \mathcal{L}(\mathcal{A})$.

In the rest of the paper, we will only consider local HMSCs. However, this is not sufficient to ensure correctness of synthesis. Let us consider the projection of $H$ in Figure 3 on all its instances given in Figure 6. A correct behavior of $H$ is shown in Figure 7-a), while a possible but incorrect behavior of the synthesized automata is shown in Figure 7-b). We can see that message $m_{4}$ sent by machine $D$ can arrive at machine $C$ while $m_{2}$ sent by machine $B$ is still in transit. According to the HMSC semantics, machine $C$ should delay the consumption of $m_{4}$ to receive message $m_{2}$ first. However, 


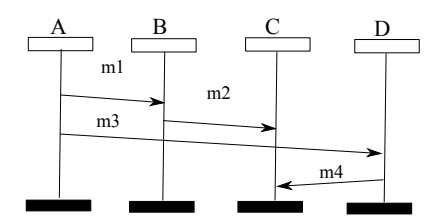

(a)

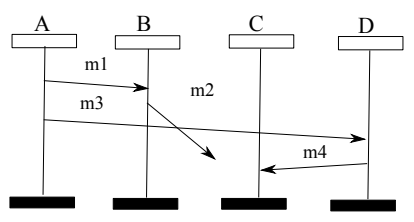

(b)

Fig. 7: a) A correct behavior of the HMSC of Fig. 3-a), and b) a possible distortion due to the loss of information on projected instances.

$C$ does not have enough information to decide to delay the consumption of $m_{4}$, and hence exhibits an unspecified behavior.

This example proves that in general, even for local HMSCs, the synthesis by projection is not correct. Problems arise when an instance does not have enough information on the sequences of choices that have occurred in the causal past of a message reception event. In some sense, the projection of an HMSC on local components breaks the global coordination between deciding instances and the other instances in the system.

Definition 10 Let $H$ be a local HMSC and $c$ be a choice node of $H$. Let $\rho$ be a cyclic path starting from $c$, and $\rho^{\prime}$ be any acyclic path starting from c. Let $H_{c}$ be the HMSC with two nodes $c, c^{\prime}$, two transitions $\left(c, O_{\rho}, c\right)$ and $\left(c, O_{\rho}^{\prime}, c^{\prime}\right)$. Let $\mathcal{A}_{c}$ be the CFSM obtained by projection from $H_{c}$. We will say that $c, \rho, \rho^{\prime}$ is a sequence-loss witness iff $\mathcal{L}\left(H_{c}\right) \neq \mathcal{L}\left(\mathcal{A}_{c}\right)$.

We will say that an HMSC is reconstructible if and only if it is local and has no sequence-loss witnesses. The class of reconstructible HMSCs was proposed in [16]. This paper also shows that it is sufficient to consider simple cycles leaving a choice to detect sequence-loss witnesses, which allows for the definition of a terminating algorithm. Furthermore, one does not have to simulate all runs of communicating automata in $\mathcal{A}_{c}$ to detect that $\mathcal{L}\left(H_{c}\right) \neq \mathcal{L}\left(\mathcal{A}_{c}\right)$. Indeed, sequence losses can be detected by checking if the sequential ordering of events along a non-deciding instance in prefix $O_{\rho} \circ O_{\rho^{\prime}}$ can be lost during projection. To avoid technical details, we will not show in this paper how the sequence losses can be found from $O_{\rho} \circ O_{\rho^{\prime}}$, but rather illustrate the approach on an example. We refer interested readers to [16] for formal details. Let us consider the example of Figure 3 -a), with a single choice node $n_{0}$, and the path $\left(n_{0}, M_{1}, n_{0}\right) .\left(n_{0}, M_{2}, n_{1}\right)$. According to the semantics of HMSCs, reception of messages $m_{2}$ and $m_{4}$ on instance $C$ should occur in this order in a correct implementation of the example. Now let us consider the automata obtained by projection of $H$ on instances, as in Figure 6. After executing $A ! B(m 1) . B ? A(m 1) . B ! C(m 2) . A ! D(m 3) . D ? A(m 3) . D ! C(m 4)$, the CFSM is in configuration $\left(L=q_{1, A} \cdot q_{0, B} \cdot q_{0, C} \cdot q_{2, D}, W=\left\{\epsilon, \ldots w_{B C}=m 2, w_{D C}=\right.\right.$ $m 4, \ldots \epsilon\})$. From this configuration, the automaton corresponding to instance $C$ can receive $m 2$, which is the expected behavior, or conversely 
receive $m 4$ which is wrong according to the choices that were performed by instance $A$. Hence $n_{0},\left(n_{0}, M_{1}, n_{0}\right),\left(n_{0}, M_{2}, n_{1}\right)$ is a sequence loss witness. This can be easily seen from $M_{1} \circ M_{2}$ : If one removes the ordering between the reception of $m 2$ and the reception of $m 4$, there is no way to infer this ordering from remaining causalities. One important fact is that synthesis by projection is correct for the subclass of reconstructible HMSCs.

Theorem 3 ([16]) Let $H$ be a reconstructible $H M S C$, and $\mathcal{A}$ be the CFSM obtained from $H$ by projection. Then, $\mathcal{L}(H)=\mathcal{L}(\mathcal{A})$.

As for local HMSCs, one can easily show that detecting if an HMSC is reconstructible is a $c o-N P$ problem. According to theorem 3 , the communicating automata synthesized from reconstructible HMSCs are correct implementations. However, we show in the next section, that all local HMSCs can be implemented with the help of additional controllers. This allows for the following synthesis approach: first check if an HMSC is reconstructible. If the answer is yes, then synthesize the CFSM by simple projection as proposed in section 4. If the answer is no, then synthesize the CFSM with their controllers, as proposed in section 5 .

\section{Implementing HMSCs with message controllers}

The class of reconstructible HMSCs shown in section 4 is contained in the class of local HMSCs. This subclass is quite restrictive (for instance, the HMSC of Figure 3-a) is not reconstructible, and hence can not be implemented by a simple projection). Note also that the difference between the languages of an HMSC and of the synthesized machines comes from the fact that some communicating automata consume a wrong message instead of waiting for the arrival of the message specified by the HMSC. In this section, we address the synthesis problem in a different setting, that is we add a local controller to each communicating machine that can tag messages and delay their delivery. As synthesis fails because of reception of messages in the wrong order, each controller will receive messages destinated to the machine it controls, and decide whether it should deliver it or delay its delivery. This decision is taken depending on additional information carried by messages, namely a vector clock. Vector clocks is a well known mechanism, and helps keeping track of global progress in distributed systems.

This new mechanism allows for the implementation of any local HMSC $H$, without syntactic restriction. The architecture is as follows: For each process, we compute an automaton, as shown in previous section by projection of $H$ on each of its instances. The projection is the same as previously, with the slight difference that the synthesized automaton communicates with his controller, and not directly with other processes. To differentiate, we will denote by $K\left(A_{i}\right)$ the "controlled version" of $A_{i}$, keeping in mind that $A_{i}$ and $K\left(A_{i}\right)$ are isomorphic machines. Then, we add to each automaton $K\left(A_{i}\right)$ a controller $C_{i}$, that will receive all communications from 
$K\left(A_{i}\right)$, and tag them with a stamp. In every automaton $K\left(A_{i}\right)$ we replace each transition of the form $\left(\left(n_{1}, M_{1}, k, n_{2}\right), p ! q(m),\left(n_{3}, M_{2}, k^{\prime}, n_{4}\right)\right)$ (respectively $\left.\left(\left(n_{1}, M_{1}, k, n_{2}\right), p ? q(m),\left(n_{3}, M_{2}, k^{\prime}, n_{4}\right)\right)\right)$ in $A_{i}$, by a transition of the form $\left(\left(n_{1}, M_{1}, k, n_{2}\right), p ! C_{p}(q, m, b),\left(n_{3}, M_{2}, k^{\prime}, n_{4}\right)\right)$ (respectively $\left.\left(\left(n_{1}, M_{1}, k, n_{2}\right), p ? C_{p}(q, m, b),\left(n_{3}, M_{2}, k^{\prime}, n_{4}\right)\right)\right)$, where $b$ indicates the branch to which the sending or the reception belongs. A controller $C_{i}$ can receive messages of the form $(q, m, b)$ from his controlled process $K\left(A_{i}\right)$. In such cases, it tags them with a clock (the contents of this clock is defined later in this section), and sends them to controller $C_{q}$. Similarly, each controller $C_{i}$ will receive all tagged messages destinated to $K\left(A_{i}\right)$, and decide with respect to its tag whether a message must be sent immediately to $K\left(A_{i}\right)$ or delayed (i.e. left intact in buffer). Automata and their controllers communicate via FIFO channels, which defines a total ordering on message receptions or sendings. Controllers also exchange their tagged messages via FIFO buffering. In this section, we first define the distributed architecture and the tagging mechanism that will allow for preservation of the global specification. We then define control automata and their composition with synthesized automata. We then show that for local HMSCs the controlled local system obtained by projection behaves exactly as the global specification (up to some renaming and projection that hides the controllers).

\subsection{Distributed architecture}

We consider the $n=|I|$ automata $\left\{K\left(A_{i}\right)\right\}_{1 \leq i \leq n}$ obtained by projection of the original HMSC on the different instances, and a set of controllers $\left\{C_{i}\right\}_{1 \leq i \leq n}$. Each communicating automaton $K\left(A_{i}\right)$ is connected via a bidirectional FIFO channel to its associated controller $C_{i}$. The controllers are themselves interconnected via a complete graph of bidirectional FIFO channels. We will refer to these connections among communicating automata as ports. A machine $K\left(A_{i}\right)$ communicates with its controller via a port $P_{i}$, and for all $i \neq j$, port $P_{i, j}$ of controller $C_{i}$ is connected to the port $P_{j, i}$ of controller $C_{j}$. This architecture is illustrated in Figure 8 for three processes $i, j, k$. This architecture is quite flexible: All the components run asynchronously and exchange messages, without any other assumption on the way they share resources, memory or processors.

\subsection{Tagging mechanism}

Vector clocks are a standard mechanism to record faithfully executions of distributed systems (see for instance $[11,24]$ ), or to enforce some ordering on communication events [27]. Usually, vector clocks count events that have occurred on each process. In the architecture that we defined, each controller maintains a vector clock that counts the number of occurrences of each branch of an execution it is aware of. 


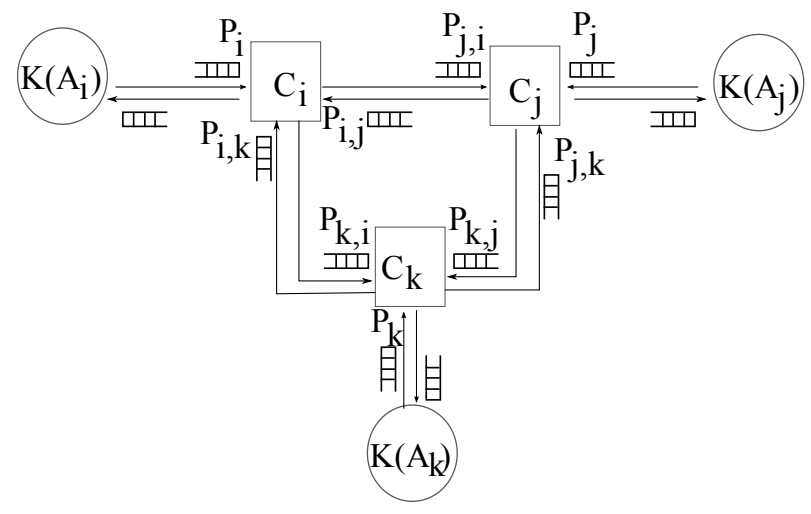

Fig. 8: The distributed controlled architecture.

To allow for faithful recording of branches chosen along an execution we have to set up a total ordering on branches of HMSCs. Let $H$ be an HMSC. We will denote by $\mathcal{B}_{H}$ the branches of $H$, and fix an arbitrary total ordering $\triangleleft$ on $\mathcal{B}_{H}$. We use this arbitrary order on branches to index integer vectors that remember the number of occurrences of branches that have occurred during an execution of an HMSC. Let us consider the example of Figure 3, that contains two branches $b_{1}=\left(n_{0}, M_{1}, n_{0}\right)$ and $b_{2}=\left(n_{0}, M_{2}, n_{1}\right)$. We can fix $b_{1} \triangleleft b_{2}$, and associate to every execution a vector $\tau$ of two integers, where $\tau\left[b_{i}\right], i \in 1,2$ represents the number of occurrences of branch $b_{i}$ in the execution.

Definition 11 (Choice clocks) $A$ choice clock of an HMSC H is a vector of $\mathbb{N}^{\mathcal{B}_{H}}$. Let $\rho=n_{0} \stackrel{M_{1}}{\longrightarrow} n_{1} \stackrel{M_{2}}{\longrightarrow} n_{2} \ldots \stackrel{M_{k}}{\longrightarrow} n_{k}$ be a path of $H$. The choice clocks labeling of $O_{\rho}$ is a mapping $\tau: E_{O_{\rho}} \longrightarrow \mathbb{N}^{\mathcal{B}_{H}}$ such that for every $i \in$ $1 . . k, e \in M_{i}, \tau(e)[b]$ is the number of occurrences of branch $b$ in $M_{1} \circ \cdots \circ M_{i}$.

Intuitively, choice clocks count the number of occurrences of each choice in a path of $H$. In the rest of this section, we will show that communicating automata and their controllers can maintain locally a choice clock along the prefix that they are executing, and that choice clocks carry all the needed information to forbid the execution of prefixes that are not in $\mathcal{L}(H)$.

The usual terminology and definitions on vectors apply to choice clocks. A vector $V_{2}$ is an immediate successor of a vector $V_{1}$ of same size, denoted $V_{1} \lessdot V_{2}$, if there is a single component $b$ such that $V_{1}[b]+1=V_{2}[b]$, and $V_{1}\left[b^{\prime}\right]=V_{2}\left[b^{\prime}\right]$ for all other entries $b^{\prime}$. We will say that vectors $V_{1}$ and $V_{2}$ are equal, denoted $V_{1}=V_{2}$, if $V_{1}[b]=V_{2}[b]$ for every entry $b$. We will say that $V_{2}$ is greater than $V_{1}$, denoted $V_{1} \prec V_{2}$, iff $V_{1}[b]=V_{2}[b]$ for some entries $b$, and $V_{1}[b]<V_{2}[b]$ for all others.

For a given path $\rho=n_{0} \stackrel{M_{1}}{\longrightarrow} n_{1} \stackrel{M_{2}}{\longrightarrow} n_{2} \ldots \stackrel{M_{k}}{\longrightarrow} n_{k}$, we will call the choice events of $O_{\rho}$ the minimal events in every $M_{i}, i \in 1 . . k$. It is rather straightforward to see that when an HMSC $\mathrm{H}$ is local, then for every path 
$\rho$ of $H$, the set of choice events in $O_{\rho}$ is totally ordered. Note also that for a pair of events $e, f$ in $O_{\rho}, \tau(e)=\tau(f)$ if and only if $e, f$ belong to the same bMSC $M_{i}$. From these facts, the following proposition is straightforward:

Proposition 2 Let $H$ be a local $H M S C, \rho$ be a path of $H$, and $\tau$ be the choice clock labeling of $O_{\rho}$. Then, $\left(\tau\left(E_{O_{\rho}}\right), \prec\right)$ is a totally ordered set.

This proposition is important: maintaining locally a consistent tagging of messages allows a controller that has two tagged messages available in two of its buffers to decide which one should be delivered first.

Definition 12 (Concerned instances) Let $b=(c, M, n)$ be a branch of an $H M S C H$. We will say that instance $p \in I$ is concerned by branch $b$ if and only if there exists an event of $M$ on $p\left(E_{M p} \neq \emptyset\right)$. Let $K \in \mathbb{N}^{\mathcal{B}_{H}}$ be a choice clock, and let $p \in I$ be an instance of $H$. The vector of choices that concern $p$ in $K$ is the restriction of $K$ to branches that concern $p$, and is denoted by $[K]_{p}$.

In the example in Figure 3, the choice clock is a integer vector indexed by $b_{1}, b_{2}$, where $b_{1}=\left(n_{0}, M_{1}, n_{0}\right)$ and $b_{2}=\left(n_{0}, M_{2}, n_{1}\right)$. Considering $M_{1}$ and $M_{2}$ as defined in Figure 2, instances $A, C$ are concerned by both branches (they are active in $M_{1}$ and $M_{2}$ ), but instance $B$ is concerned only by $b_{1}$ and instance $D$ is concerned only by $b_{2}$.

For a given instance $i \in I$, the controller $C_{i}$ associated with the projected automaton $K\left(A_{i}\right)$ will receive the messages sent by $K\left(A_{i}\right)$ and by the other controllers. Messages exchanged between the automata and the controllers are triples $(j, m, b)$ where $j \in I$ is the destination automaton, $m \in C$ is the message name, and $b$ the branch in which the sending event has occurred. In other words, in our controlled architecture, an automaton executes $p ! C_{p}(q, m, b)$ instead of $p ! q(m)$. The messages exchanged between controllers are tagged and represented by pairs $(m, \tau)$ where $m$ is a message name and $\tau \in \mathbb{N}^{\mathcal{B}_{H}}$ a choice vector. In addition, the controller $C_{i}$ maintains several local variables:

$-\tau_{i} \in \mathbb{N}^{\mathcal{B}_{H}}$, its locally known choices vector. It is initialized to the null vector, and updated upon consumption of incoming messages.

- numEvt, which counts the remaining number of communication events of the instance $i$ to be treated in the current branch that is being processed.

- Rec is a sequence of reception events. numEvt and Rec are initialized with constant values (that depend on the chosen branch) when dealing with the first event of a branch on process $i$.

- currentb, which memorizes the branch of $H$ that is currently executed by process $i$.

In the rest of the paper, we will denote by $\pi_{i}(M)$ the sequence of events obtained by projection of $M$ on instance $i \in I$, and by $\pi_{i, ?}(M)$ the restriction of this sequence to receptions. For a sequence of events $w$, we will 
denote by $\operatorname{tail}(w)$ the sequence of events obtained by removing the first event from $w$, that is if $w=a . v$, then $\operatorname{tail}(w)=v$. The generic algorithm for a controller $C_{i}$ is composed of two rules, which are always active (see Algorithm 2). Rule 1 applies to communications from $K\left(A_{i}\right)$ to $C_{i}$. First case corresponds to minimal events controlled by the projected automaton $K\left(A_{i}\right)$. When dealing with the first event of the bMSC (branch $b$ ) to be processed, the only role of the controller is to compute the tag (increment of the corresponding component of $\tau_{i}$ ) and to initialize the variables numEvt and Rec. The currently processed branch is stored in variable currentb. The other case deals with communications from $K\left(A_{i}\right)$ that are not choices of $K\left(A_{i}\right)$. These events are generated in correct order by construction of the projection.

The second rule applies for every port $P_{i, j}, j \neq i$, and aims at controlling the order of the different receptions of messages arriving in the buffers between each controller $C_{j}, j \in I \backslash\{i\}$ and controller $C_{i}$. This is the main objective of the controller. Note that these messages arrive in a distinct buffer for each neighbor controller. There are three cases:

- The first case occurs when a branch of $H$ has already been started, that is a controller $C_{i}$ has received (i.e. consumed) a message indicating the choice performed by the deciding instance of this branch, and a valid message arrives. In this situation, all the components concerning $K\left(A_{i}\right)$ of the current tag $\tau_{i}$ and of the tag $\tau$ labeling the incoming message must be equal, and this incoming message must be the next expected message (i.e. the next reception in $R e c$ ) in the currently executed branch. Then the message can be consumed by $C_{i}$ and forwarded to $K\left(A_{i}\right)$. The fact that there is only one FIFO channel between the controller $C_{i}$ and the projected automaton $K\left(A_{i}\right)$ ensures the correct order of receptions on this automaton.

- The second case is when the incoming message is the first communication signaling a new choice. The controller then checks if the received message defines the next branch of $H$ that must be executed by $K\left(A_{i}\right)$. This is done by verifying if the received tag is the next tag to be treated (considering only the components that concern $K\left(A_{i}\right)$ ), that is $\left[\tau_{i}\right]_{i} \lessdot[\tau]_{i}$. In that case, the current tag can be updated. The current branch is retrieved by considering the component that differs between $[\tau]_{i}$ and $\left[\tau_{i}\right]_{i}$. Then the remaining number of events that should be executed within this branch (the number of events on the instance $i$ in the bMSC of the current branch, minored by one) is set, as well as the expected sequence of receptions, before transmission of the message to $K\left(A_{i}\right)$.

- The third case applies when none of the above situations hold, that is the incoming message on port $P_{i, j}$ can not yet be consumed, either because it is not the next reception expected (another reception on another port should occur before this one) or the incoming message signals that a new choice has been started, but more events must occur before consuming it. In such case, the controller does nothing, and waits for other messages on other ports. 
The algorithm 2 executed by every controller is presented next page.

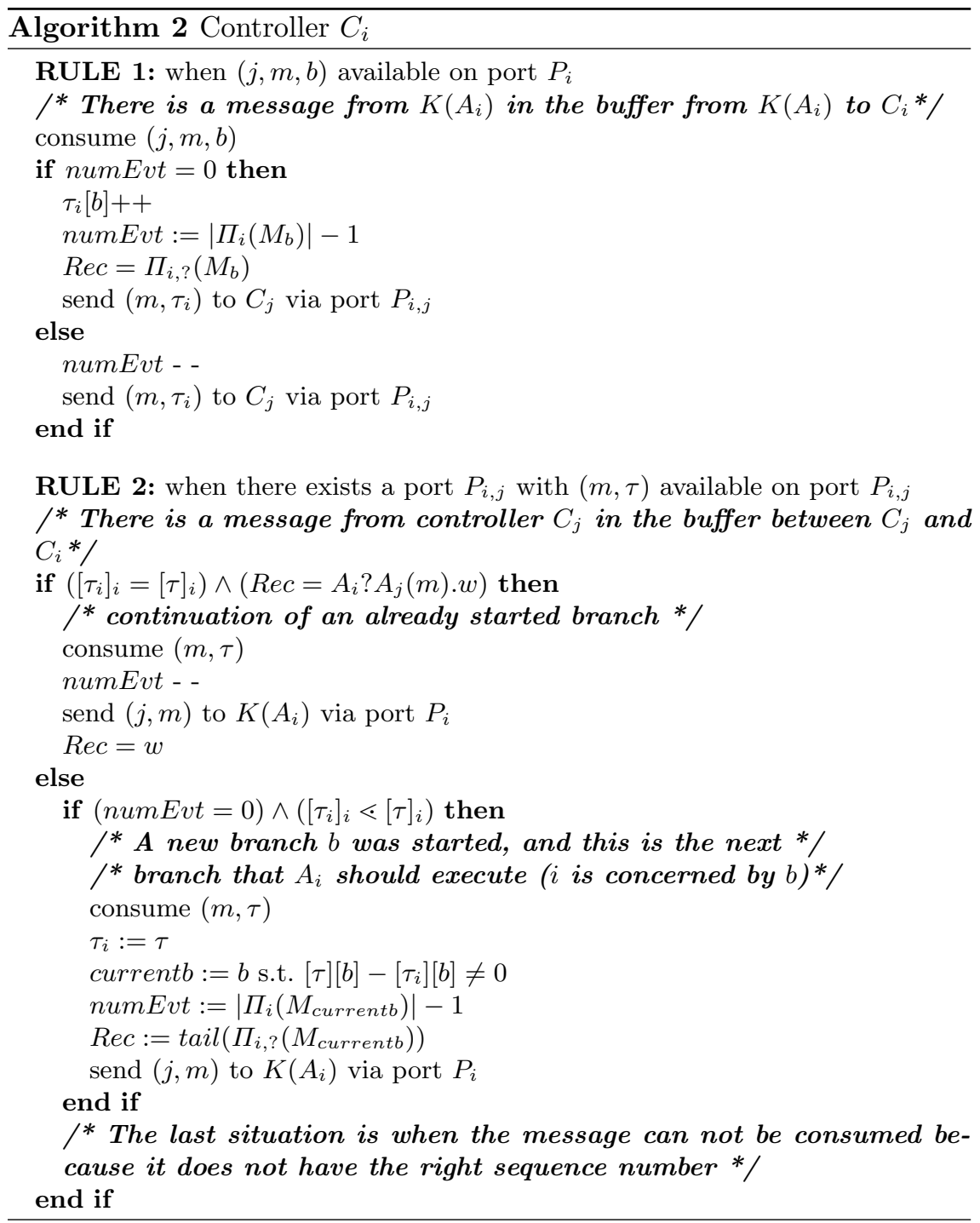

Now that we have defined controlled automata and their controllers, we can define formally how they compose. Recall that $K\left(A_{i}\right)$ is a finite state machine with the same states as $A_{i}$, but in which each transition $\left(q, i ! j(m), q^{\prime}\right)$ is replaced by a transition $\left(q, i ! C_{i}(j, m, b), q^{\prime}\right)$ (where $b$ denotes the name of the branch currently executed by $A_{i}$, and each transition $\left(q, i ? j(m), q^{\prime}\right)$ is replaced by a transition $\left(q, i ? C_{i}(j, m), q^{\prime}\right)$. Each controller $C_{i}$ is not a communicating automaton, but yet it is a machine that sends and receives messages. The composition $K\left(A_{i}\right) \mid C_{i}$ of a machine with its controller is a 
pair of communicating machines with a FIFO buffer from $K\left(A_{i}\right)$ to $C_{i}$, and another from $C_{i}$ to $K\left(A_{i}\right)$. Then, the composition of controlled machines $\|_{i \in I}\left(K\left(A_{i}\right) \mid C_{i}\right)$ is the union of all $K\left(A_{i}\right) \mid C_{i}$, with communication buffers from each $C_{i}$ to each $C_{j}$, for $i \neq j$ in $I$. Note that $K\left(A_{i}\right)^{\prime} s$ communicate only with their controllers. This composition is illustrated in Figure 8, where the depicted architecture is $\left(K\left(A_{i}\right) \mid C_{i}\right)\left\|\left(K\left(A_{j}\right) \mid C_{j}\right)\right\|\left(K\left(A_{k}\right) \mid C_{k}\right)$. At this point, let us note that our controlled implementation is not a CFSM anymore. Note that our controllers are defined with several lines of code, but that they simply recall a local state plus an increasing vector of integers. The number of local states that a controller can record is finite (they are simply the states of the finite automaton obtained by projection on the instance). So, the infinite part of the controller only comes from the vector. Another light modification with respect to standard communicating machines is that the controller needs to read messages without consuming them. Note however, that variables, message reading, etc. are allowed in extended state machine models such as SDL [19]. Considered individually, process descriptions obtained after controlled synthesis are represented by an automaton plus its controller. However, the correctness result presented hereafter shows that the synthesis does not change the individual behavior of an instance, which remains regular. The major difference between the standard architecture and the controlled one is that the controlled automata 'simulate' the original specification (controllers are allowed to play additional hidden sequences of events before delivering a message), while the automata obtained by projection in the standard synthesis framework of section 4 have to play exactly the sequences of events described by the original HMSC to be a correct implementation.

\subsection{Correctness of controlled synthesis}

Let us show correctness of the synthesis with local controllers. Of course, adding controllers to the system means adding the controllers' actions to the executions. Hence, we can not require that $\mathcal{L}(H)=\mathcal{L}\left(\|_{i \in I}\left(K\left(A_{i}\right) \mid C i\right)\right.$ anymore. We propose another notion of correctness, namely language equality up to abstraction of controllers. Abstraction erases controllers' actions, and considers communications $(q, m, b)$ from a process $p$ to his controller as a communication of a message $m$ from $p$ to $q$.

Definition 13 Let $O=(E, \leq, t, \phi, \mu)$ be a prefix in $\mathcal{L}\left(\|_{i \in I}\left(K\left(A_{i}\right) \mid C i\right)\right.$. The restriction of $O$ to non-control events is a restriction of $O$ to events located on $K\left(A_{i}\right)$ 's. We will denote this restriction by $\operatorname{Unc}(O)$. The uncontrolling of $O=(E, \leq, t, \phi, \mu)$ is a renaming function $R u()$ that replaces communications to and from the controller of a process by direct communications with the process concerned by the sent/received message, and builds the message mapping. $R u(O)=\left(E, \leq, t^{\prime}, \phi, \mu^{\prime}\right)$, where $t^{\prime}(e)=p ! q(m)$ if 
$t(e)=K\left(A_{p}\right) ! C_{p}(m, q, c), t^{\prime}(e)=p ? q(m)$ if $t(e)=K\left(A_{p}\right) ? C_{p}(m, q)$, and $t^{\prime}(e)=t(e)$ otherwise. Function $\mu^{\prime}$ maps the $i^{t h}$ sending from $p$ to $q$ with the $i^{\text {th }}$ reception on $q$ from $p$ for every pair of processes.

Note that for a prefix $O$ in $\mathcal{L}\left(K\left(A_{i}\right) \mid C i\right)$ (i.e. located on a single instance), the message mapping in $\operatorname{Unc}(O)$ is an empty relation.

Theorem 4 Let $H$ be an $H M S C$, and let $\|_{i \in I} K\left(A_{i}\right) \mid C_{i}$ be its controlled synthesis. Then, $\operatorname{Ru}\left(\operatorname{Unc}\left(\mathcal{L}\left(\|_{i \in I} K\left(A_{i}\right) \mid C_{i}\right)\right)\right) \stackrel{i \in I}{=} \mathcal{L}(H)$.

Proof sketch: We want to show that the original specification given as an HMSC and the synthesized controlled machines exhibit the same behaviors. We proceed in several steps. We first show that in the synthesized machines, all choices (i.e. events corresponding to the first event of some bMSC) are causally ordered in any execution of the network of synthesized machines and controllers. We then show that for every configuration of an HMSC $H$ reachable after an execution $O$, there exists a finite set of configurations of the synthesized machines reachable by observing the same execution. The last steps of the proof show inclusion of specification and implementations languages in both directions by contradiction. Supposing that there exists a configuration of $H$ reached after executing a prefix $O$ that allows firing of an event $a$ but that there exists no corresponding configuration of the CFSM reachable after $O$ that allows $a$ leads to a contradiction. We consider each type of events for $a$ and show that allowing $a$ in one language but not in the other contradicts either the fact that $O$ is a prefix of both the original specification and the synthesized language, or the fact that choices are ordered. A complete proof of this theorem can be found in a research report available at http://people.rennes.inria.fr/Loic.Helouet/Papers/RR-7597.pdf $\square$

This result shows correctness of synthesis up to renaming, and erasing of controllers' moves. As a consequence, the behavior of an instance $i \in I$ in an HMSC, and the behavior of the CFSM $K\left(A_{i}\right)$ are isomorphic. Hence, even after adding infinite controllers, the behaviors of processes remains regular.

\section{Use case}

In order to illustrate the way our algorithm works we study controlled synthesis from an example HMSC describing a simple transmission protocol based on the Morse code. This example is shown in Figure 9. In this figure, we indicate in italic an index associated to each branch of the HMSC (for instance transition $\left(n_{0}, M 0, n_{1}\right)$ is branch $\left.b 1\right)$. One can immediately notice that this HMSC is local. However, it is not reconstructible, and the respective ordering between messages zero and one can be lost. In this example, process $A$ wants to transmit Morse coded information to process $B$. The protocol can be decomposed into several phases. If $A$ has nothing to send, 
it indicates it to the coder which acknowledges that there will be no transmission via a message Leave (bMSC M6). To send a message, $A$ requires a connection to $B$ via the Morse Coder $C$ (bMSC $M 0$ ). Once the connection is established $A$ can leave the transmission (bMSC M5), or send the information to the Morse Coder $C$ (bMSC $M 1$ ). Then, $C$ translates the information received from $A$ into a sequence of binary digits (0's and 1's) that represent respectively the dots and the dashes of the Morse code. Then $C$ can send the elements of this sequence to $B$ via two channels: All the 0s are sent via the channel chan 0 (bMSC M2) and all the 1s are sent via the channel chan 1 (bMSC M3). Once the coded information is completely transmitted to $B$, the process $C$ sends an acknowledgment to the process $A$ that can choose either to send a new piece of data or to close the connection.

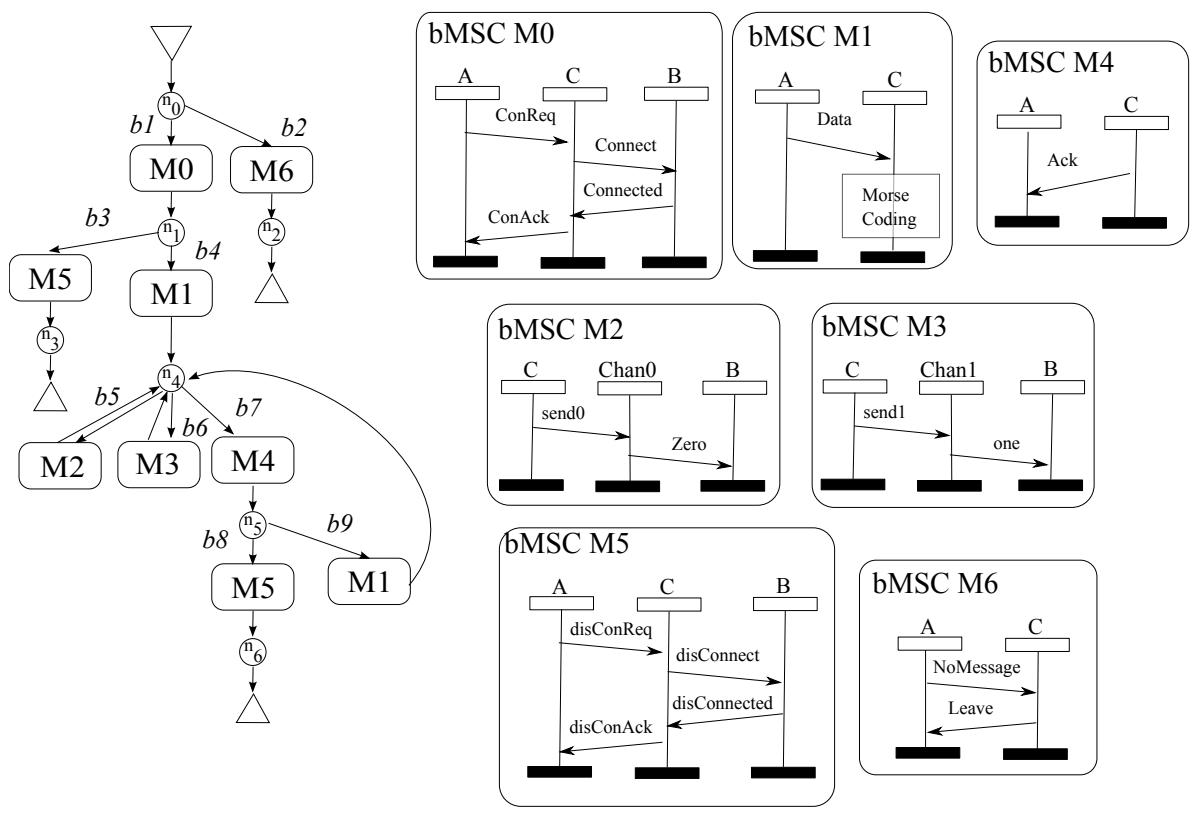

Fig. 9: A simple transmission protocol based on Morse code

Figure 10 shows the projection of the HMSC of Figure 9 on all the instances of the system. As this HMSC is not reconstructible, we must use the controlled synthesis technique described in section 5 . Hence, each process interacts with its controller. We will give a number to each process going from 0 to 4 associated respectively to the processes $A, B, C, C h a n 0$ and Chan1, and call Cont $_{x}$ the controller attached to instance $x$. Let us explain the structure of the exchanged messages between the automata and their controllers: For example $A$ wants to send to $C$ the message ConReq (hence choosing branch $b 1$ in the HMSC), then $A$ sends to its controller the message 


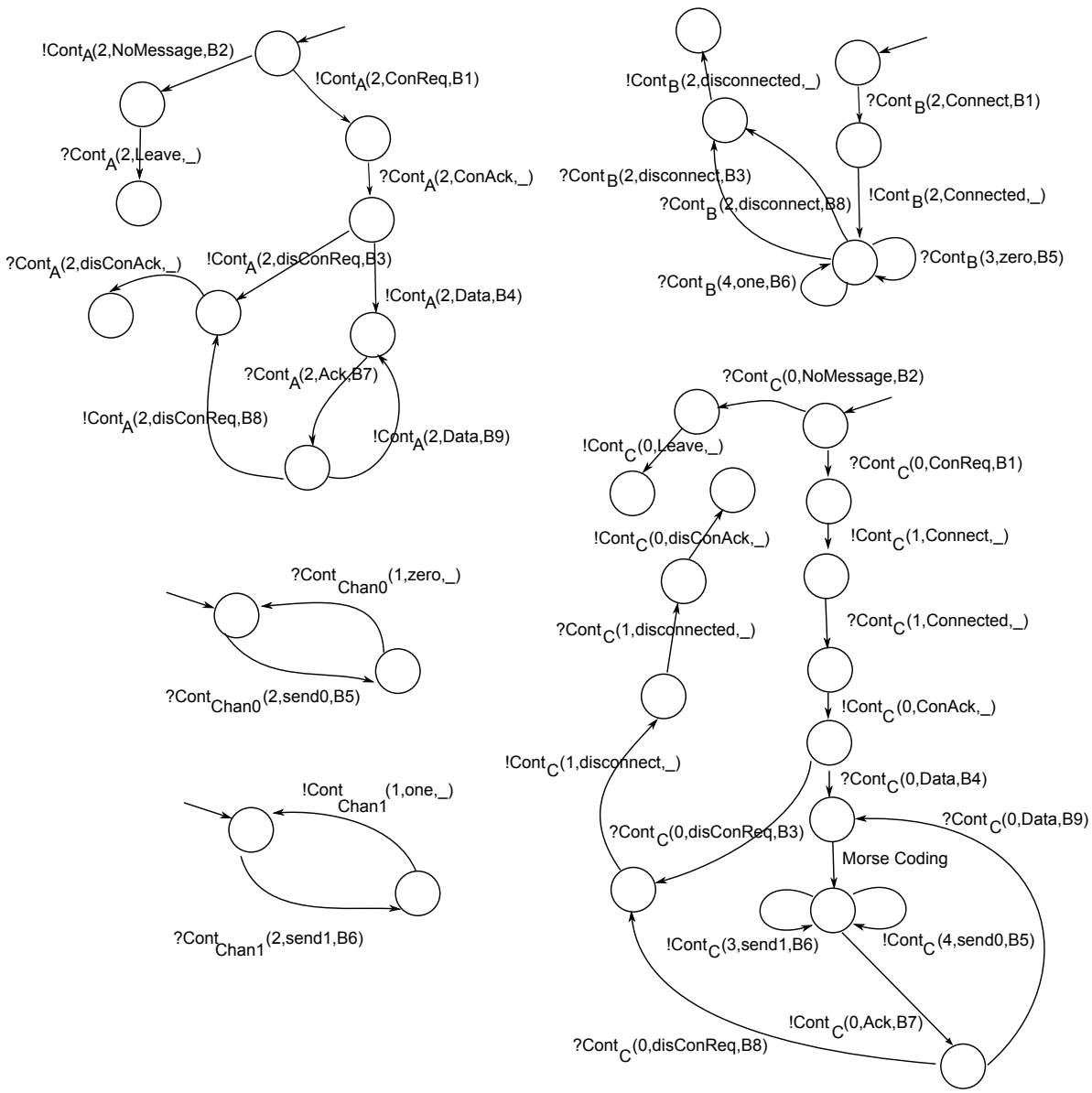

Fig. 10: The CFSM obtained by projection of the HMSC Figure 9

$(2$, ConReq,$b 1)$ that corresponds to the $(i, m, j)$ structure described in the algorithm. The 2 in the message corresponds to the destination process, so when the controller receives the messages it knows to which instance controller it should be forwarded (the process number 2 is $C$ ). ConReq represents simply the data part of the message that should be sent by the controller, $b 1$ means that the process $A$ wants to start a new scenario (or bMSC). So, upon reception of this message, the controller $C_{\text {ont }} A$ have to update its local tag. The new tag will then be concatenated to the message that will be sent by $\mathrm{Cont}_{A}$. The Morse example can be controlled using tags in $\mathbb{N}^{9}$, as there are 9 different branches in our HMSC. Note that the tagging mechanisms are not meant to be written by a user, but rather automatically generated by a tool. The tool SOFAT [15], a scenario platform developed at IRISA has been extended to allow such generation. 


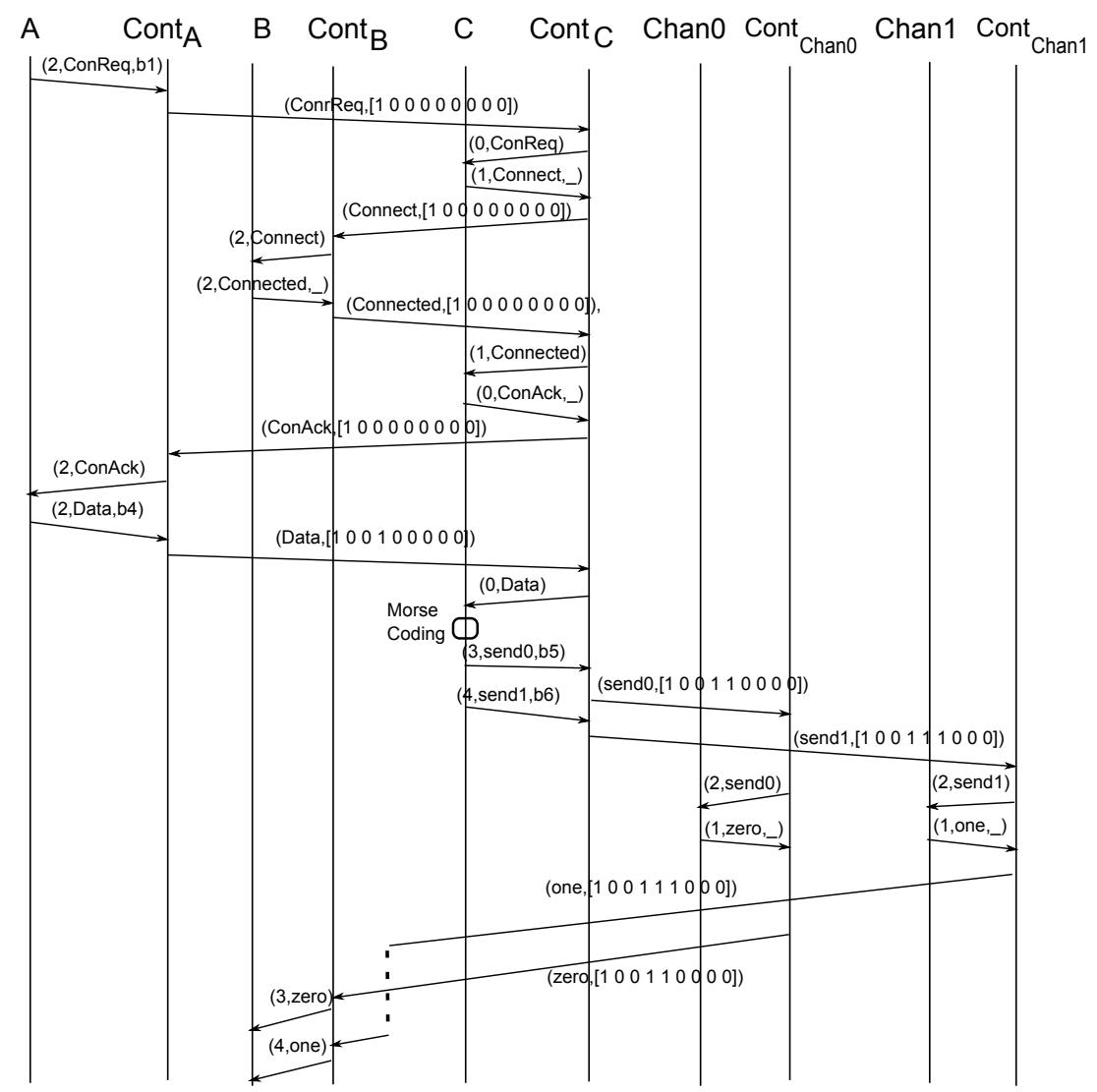

Fig. 11: An execution of the CFSM of Figure 10 with their controllers

An example of execution for the path $n_{0} \stackrel{M 0}{\longrightarrow} n_{1} \stackrel{M 1}{\longrightarrow} n_{4} \stackrel{M 2}{\longrightarrow} n_{4} \longrightarrow$ $n_{4} \stackrel{M 3}{\longrightarrow} n_{4}$ is shown in Figure 11. In $M 0$ the connection is established between the processes $A$ and $B$ via $C$, then process $A$ sends to $C$ the data to be coded and sent to process $B$. $C$ codes the data: In the example, it is coded as a sequence of two bits: 01 . Instance $C$ chooses branch $b 5$ (bMSC M2) then branch $b 6$ (bMSC M3). A difference in the performance between chan 0 and $\operatorname{chan} 1$ causes a delay and a message overtaking in the diagram: The message one is received by $\operatorname{Cont}_{B}$ (the controller associated with $B$ ) before the message zero. Hence, Cont $_{B}$ has a message of the form (one, $\tau$ ) in its buffer collecting messages from Cont $_{\text {Chan } 1}$, and no message in its buffer collecting messages from Cont $t_{\text {Chan } 0}$. Then, Cont $_{B}$ compares the tag $\tau=$ [100111000]) of this message with its local tag $\tau_{B}=$ [100000000] (set to this value after the execution of the bMSC $M 0$ as $B$ is an active instance of $M 0)$. The projection $[\tau]_{B}=[10110]$ of the received tag on the branches concerning $B$ is not an immediate successor of $\left[\tau_{B}\right]_{B}=[10000]$, 
so the controller delays the delivery of the message one to the automaton $B$, and leaves the message in its buffer. When $\operatorname{Cont}_{B}$, receives the message zero with tag $\tau^{\prime}=[100110000]$ in its buffer from Cont $_{C 0}$, as $\left[\tau^{\prime}\right]_{B}=[10100]$ is the immediate successor of $\left[\tau_{B}\right]_{B}=[10000]$, the message is forwarded to $B$ and Cont $_{B}$ updates its local tag to [100110000]. When Cont $_{B}$ compares again the tag of the delayed message one with its local tag, $[\tau]_{B}=[10110]$ is the immediate successor of $\left[\tau_{B}\right]_{B}=[10100]$ so Cont $_{B}$ forwards the message one to $B$ and updates its local tab to [100111000]. Hence, a correct ordering in the reception of messages zero and one on instance $B$ is ensured by the tagging mechanisms despite the message crossing.

\section{Related work}

Protocol synthesis is not a new problem, and stems from the 80's [7]. The addressed problem is: Given a high level specification with global control (the decisions taken in the system may consider a global state of all agents), how to produce an implementation, i.e. a set of independent (hence with local control only) machines that exhibit the same behaviors as the original specification? This problem has been addressed with many specification and implementation formalisms. For a generic survey on protocol synthesis, we refer interested readers to [28]. Note that synthesis may not be achievable.

In recent years, a lot of attention has been paid to synthesis from scenario notations. Synthesis from scenarios can be easier to implement when the chosen formalism includes indications on communications and on the distribution of tasks among agents. The problem has been addressed for MSCs [1,16], LSCs [14], sequence diagrams [32], and other scenario-based models. We do not claim for exhaustiveness in our bibliography, and refer interested readers to $[3,22]$ for surveys on synthesis from scenarios. These surveys compare and classify many approaches, and we next classify our technique according to the comparison criteria provided in these surveys.

Liang et al [22] compare the synthesis approaches according to the source formalism, the intended use (analysis or code generation), the support for composition operators and parallelism. Our approach uses High-level MSCs as input language, and supports composition operators such as loops, sequence, and choices. We consider parallelism among agents, but there is no support of parallel frames. The reasons for this restriction are discussed in section 2: Parallel frames introduce non-determinism leading to incorrect synthesized behaviors, and may force implementations to have an unbounded number of control states. The intended use of our technique is mainly code generation. Other interesting criteria address the target model, which can be with global or local control, the degree of automation, and tool support. The synthesis proposed in this paper derives local finite state machines, which are controlled asynchronously by machines able to delay some messages according to some information about ordering of messages coded as counters. The technique is fully automated, and is implemented in our 
SOFAT tool [15]. Last, Liang et al check if the synthesis technique checks correctness and completeness of the synthesized model. Our synthesis approach is not concerned by these criteria, as the derived CFMS are correct and complete by construction, i.e. they exhibit exactly the same behaviors as the original description.

Amyot et al [3] use some criteria of [22], and introduce several other such as component focus, which considers whether the distribution of behaviors is detailed in the specification formalism, hiding i.e. the specification formalism considers internal behavior of the modeled system as a black box or allows description of internal details. In addition, Amyot et al consider representation issues i.e. whether the specification formalism is graphical or textual, and ordering issues, i.e. whether concurrency is made explicit in the formalism, time (does the scenario model and the synthesis approach address time issues?), abstraction (can the scenario model represent generic behaviors), identity (the ability to define generic scenarios involving groups of agents rather than precisely identified ones), and dynamicity (the ability to change the behavior of agents at runtime). Clearly, the HMSCs considered in our approach emphasize distribution of actions over agents, and allows for description of internal behaviors using internal actions. HMSCs are both a graphical and textual language. Though the whole HMSC language allows for abstraction, time (use of timers and expression of time constraints on scenarios), decomposition, dynamic process creation, or definition of abstract instances, we do not address these features of the language in our synthesis solutions. The most important and interesting (but also the most difficult) issues to address using such techniques are certainly time and dynamic process creation. However, defining time constraints, for instance can completely change the interpretation of a specification, and even make it inconsistent. Furthermore, time constraints involving events located on distinct instances (for instance the maximal delay allowed between the sending of a message and its reception) are hard to implement. Dynamicity is also hard to address, as there is a lack of formal distributed models allowing dynamic process creation. A first attempt to propose a dynamic communicating automaton model appeared in [8], but the proposed model must be highly non-deterministic in order to implement dynamic MSCs.

Let us now compare our approach with some former synthesis works based on HMSC projection. Most of these works propose solutions for syntactic subclasses of HMSCs only, and usually local HMSCs. We have shown in this paper that working with local HMSCs is not sufficient to guarantee a correct synthesis. Indeed, the machines synthesized by the MSC2SDL tool [1] or the MOST tool [23] frequently allow for more behaviors than the original specification. To solve this problem, [16] introduced reconstructible $H M S C s$ and showed that synthesis by projection is correct for this subclass. The solution in [12] uses local HMSCs, and furthermore requires that all processes of the HMSC are active (i.e. send or receive a message) in all branches. The approach in [5] considers regular HMSC specifications, that is a subclass of HMSCs with the expressive power of finite automata, and 
synthesizes a correct target model. Other works allow the implementation to deadlock [25] and consider that deadlocked runs are not part of the implemented language. In our approach, we have ruled out this possibility, and considered that every initiated run had to be considered as a behavior of the synthesized machines, which seems more realistic. Our approach synthesizes a correct distributed implementation for the whole class of local HMSCs. Correctness is an improvement with respect to [1,23], and completeness an improvement with respect to [12]. Many synthesis approaches proposed these last 10 years assume a synchronous semantics of HMSCs (usually by considering synchronous communications among instances, or synchronization among instances at the end of each bMSC), and take finite state machines, or statecharts variants as target language. The work in [29] assumes synchronous communications in bMSCs, and defines the semantics of HMSCs as a parallel (and synchronous) composition of finite state machines associated to instances. As a result, the synthesized specification is a finite automaton. The work in [21] synthesizes RoomCharts (a variant of statecharts) as target language, and hence assumes a synchronous semantics of HMSCs. The synchronous approach is well adapted to contexts where instances are seen as components of a synchronous system. Synthesizing finite objects then allows for standard model-checking techniques. Again, we refer interested readers to surveys $[3,22]$ for a more exhaustive list of synthesis approaches with statecharts variants as target language. On the other hand, our approach considers the standard asynchronous semantics of HMSCs [18], and allows for the synthesis of independent components, that communicate asynchronously. The semantics of the synthesized machines is not always finite state.

\section{Conclusion}

We have considered the synthesis problem for HMSCs. Synthesis of CFSMs by a simple projection mechanism is correct for local and reconstructible HMSCs. We have proposed a new solution to synthesize correct implementation for local HMSCs that are not reconstructible: Additional controllers simply tag messages and delay them to ensure correct ordering of message receptions. We think that the class of local HMSCs is a good compromise between the abstraction that is required in a specification formalism, and the preciseness that is needed for a model to be implementable. Indeed, imposing local choices avoids considering in the synthesis some heavy synchronization mechanisms among instances to ensure that distant processes behave according to the same chosen scenario. The class of local HMSCs seems expressive enough to model many interesting protocols, and furthermore, locality of HMSCs is decidable. The synthesis algorithms have been implemented in our tool SOFAT [15], to generate a formal description of the CFSM from an HMSC, Promela code, or even java code for all the instances and controllers needed in the system. 
This paper focuses on local HMSCs, but solutions still exist to synthesize correct (up to some abstraction) machines from non-local HMSCs. Indeed, a non-local HMSC can be made local by adding new synchronization messages. In the future, we plan to explore how to integrate the computation of optimal and efficient synchronization in a design methodology. The integration of data is another challenging aspect. The techniques proposed in this paper only address the control flow in a high-level description, and do not consider data. Inserting manipulation of local data in the internal actions of processes can be done easily by mixing the language of bMSCs with a data manipulation language. The code attached to actions can then be copied as it is in the generated code, which does not really impact the synthesis process. However, if data are shared and used to guard choices in HMSCs, the projection technique does not necessarily work, and additional synchronization and consistency mechanisms are needed to ensure that the synthesized processes work with the same data values.

Time issues are also complex to handle. If we consider for instance as an input model a time-constrained MSC [2], synthesizing a correct model means synthesizing machines that meet all the time constraints expressed in the specification. This imposes in particular that controllers should also play the role of timed schedulers. In such a context, using timed languages equality as a notion of correctness for synthesis seems too constraining, and one should probably restrict to timed languages inclusion.

A more technical perspective is to optimize our algorithm to reduce the size of tags. A first challenge is to reduce the number of branches that a controller have to consider. A first intuition is that only non-reconstructible choices should be remembered, but yet this has to be demonstrated. A second possibility is that all branches of a choice need not be remembered if they can not be used as witnesses for non-locality. Another aspect is to try to bound the integers used in choice clocks. This could be done by general decrease of all entries of clocks when every entry has exceeded some threshold $k$, but with additional synchronization among controllers.

Last, to keep the construction of CFSM simple, we have assumed that communications were FIFO, and as a consequence that the HMSCs considered did not contain message overtaking. The standard HMSCs allow explicit specification of message crossing inside a bMSC (but not of two messages from distinct bMSCs). Extending our techniques to models that allow message overtaking in bMSCs should be easy, as it needs only to allow a bounded number of lookaheads in FIFO buffers. Such possibility was for instance proposed in the MSC2SDL tool [1]. One fact worth mentioning again is that the controllers are purely asynchronous, which leaves a lot of freedom to choose a particular architecture. In a real implementation, one may suppose that a process and its controller are implemented on the same machine, but this is not mandatory. Controllers are designed to need as little information as possible to ensure that the processes they control are always executing a valid run of the specification: Each process executes its task as defined in the projection of the specification, and controllers ensure 
coordination. In the future, we would like to study whether asynchronous controllers can in addition enforce properties such as boundedness of buffers, avoidance of a given configuration, etc.

Acknowledgments: We wish to thank anonymous reviewers for careful reading of this paper, and for numerous comments which helped improving it.

\section{References}

1. M. Abdalla, F. Khendek, and G. Butler. New results on deriving SDL specifications from MSCs. In SDL Forum, pages 51-66, 1999.

2. S. Akshay, M. Mukund, and N.K. Kumar. Checking coverage for infinite collections of timed scenarios. In CONCUR'07, pages 181-196, 2007.

3. D. Amyot and A. Eberlein. An evaluation of scenario notations and construction approaches for telecommunication systems development. Telecommunication Systems, 24(1):61-94, 2003.

4. D. Amyot and G. Mussbacher. User requirements notation: the first ten years, the next ten years. Journal of software, 6(5):747-768, May 2011.

5. N. Baudru and R. Morin. Synthesis of safe message-passing systems. In FSTTCS, pages 277-289, 2007.

6. H. Ben-Abdallah and S. Leue. Syntactic detection of process divergence and non-local choice in Message Sequence Charts. In Proc. of TACAS'97, volume 1217 of LNCS, pages 259 - 274, April 1997.

7. G. Bochmann and R. Gotzhein. Deriving protocol specifications from service specifications. In Proc. of the ACM SIGCOMM conference on Communications architectures 83 protocols, pages 148-156, 1986.

8. B. Bollig and L. Hélouët. Realizability of dynamic MSC languages. In Proc. of CSR (Computer Science in Russia), volume 6072 of LNCS, pages 48-59. Springer, 2010.

9. D. Brand and P. Zafiropoulo. On communicating finite state machines. Technical Report RZ1053, IBM Zurich Research Lab, 1981.

10. B. Caillaud, P. Darondeau, L. Hélouët, and G. Lesventes. HMSCs en tant que spécifications partielles et leurs complétions dans les réseaux de Petri. Research Report RR-3970, INRIA, 2000.

11. C. Fidge. Logical time in distributed computing systems. IEEE Computer, 24(8):28-33, August 1991.

12. B. Genest, A. Muscholl, H. Seidl, and M. Zeitoun. Infinite-state high-level MSCs: Model-checking and realizability. In ICALP, volume 2380 of $L N C S$, pages 657-668, 2002.

13. Object Management Group. UML 2.0 : Unified Modeling Language. 2005.

14. D. Harel and H. Kugler. Synthesizing state-based object systems from lsc specifications. Int. J. Found. Comput. Sci., 13(1):5-51, 2002.

15. L. Hélouët, R. Abdallah, and D. Bhatia. SOFAT : Scenario formal analysis toolbox. 2011. www.irisa.fr/distribcom/Prototypes/SOFAT/.

16. L. Hélouët and C. Jard. Conditions for synthesis of communicating automata from HMSCs. In 5th International Workshop on Formal Methods for Industrial Critical Systems (FMICS), 2000.

17. J.E. Hopcroft and J.D. Ullman. Introduction to Automata Theory, Languages and Computation. Addison-Wesley, 1979. 
18. ITU-T. Z.120 : Message sequence charts (MSC). Technical report, International Telecommunication Union, 1998.

19. ITU-T. Z.100 : Specification and description language (SDL). Technical report, International Telecommunication Union, 2011.

20. ITU-T. Z.150 : User requirements notation (URN) - language requirements and framework. Technical report, International Telecommunication Union, 2011.

21. S. Leue, L. Mehrmann, and M. Rezai. Synthesizing software architecture descriptions from message sequence chart specifications. In ASE, pages 192195, 1998.

22. H. Liang, J. Dingel, and Z. Diskin. A comparative survey of scenario-based to state-based model synthesis approaches. In Proc. of SCESM '06: the 2006 International Workshop on Scenarios and State Machines: Models, Algorithms, and Tools, pages 5-12, 2006.

23. N. Mansurov and D. Zhukov. Automatic synthesis of sdl models in use case methodology. In SDL Forum, pages 225-240, 1999.

24. F. Mattern. Time and global states of distributed systems. in Proc. Int. Workshop on Parallel and Distributed Algorithms , Bonas, France , North Holland, pages 215-226, 1988.

25. M. Mukund, K.N. Kumar, and M. Sohoni. Synthesizing distributed finitestate systems from MSCs. In CONCUR, pages 521-535, 2000.

26. A. Muscholl, D. Peled, and Z. Su. Deciding properties for Message Sequence Charts. In FoSSaCS, volume 1378 of LNCS, pages 226-242, 1998.

27. M. Raynal, A. Schiper, and S. Toueg. The causal ordering abstraction and a simple way to implement it. Inf. Process. Lett., 39(6):343-350, 1991.

28. K. Saleh. Synthesis of communications protocols: an annotated bibliography. SIGCOMM Comput. Commun. Rev., 26:40-59, 1996.

29. S. Uchitel and J. Kramer. A workbench for synthesising behaviour models from scenarios. In ICSE, pages 188-197, 2001.

30. S. Uchitel, J. Kramer, and J. Magee. Detecting implied scenarios in Message Sequence Chart specifications. In ESEC / SIGSOFT FSE, pages 74-82, 2001.

31. S. Uchitel, J. Kramer, and J. Magee. Incremental elaboration of scenariobased specifications and behavior models using implied scenarios. ACM Trans. Softw. Eng. Methodol., 13(1):37-85, 2004.

32. T. Ziadi, L. Hélouët, and J-M Jézéquel. Revisiting statechart synthesis with an algebraic approach. In ICSE, pages 242-251, 2004. 